\title{
Optics
}

\section{Optical nonlinearity of dielectric nanocomposite}

\author{
Vladimir P. Dzyuba ${ }^{1}$, Yurii N. Kulchin ${ }^{1}$, Sergey I. Pokutnyi ${ }^{2}{ }^{*}$ \\ ${ }^{1}$ Institute of Automation and Control Processes FEB RAS, Russia, 5 Radio Str., 690041 Vladivostok, Russia \\ ${ }^{2}$ Chuiko Institute of Surface Chemistry, National Academy of Sciences of Ukraine, Kyiv, Ukraine
}

Email address:

vdzyuba@iacp.dvo.ru (V. P. Dzyuba), Pokutnyi_Sergey@inbox.ru (S. I. Pokutnyi)

\section{To cite this article:}

Vladimir P. Dzyuba, Yurii N. Kulchin, Sergey I. Pokutnyi. Optical Nonlinearity of Dielectric Nanocomposite. Optics. Special Issue: Optics and Spectroscopy of the Charge Carriers and Excitons States in Quasi - Zero - Dimensional Nanostructures.

Vol. 3, No. 6-1, 2014, pp. 22-37. doi: 10.11648/j.optics.s.2014030601.14

\begin{abstract}
In paper, being based upon the results of our works deals with experimental and theoretical studies of physical characteristics of nanoparticles responsible for specific optical properties of dielectric nanocomposites. During theoretical description and explanation of experimental data obtained, we considered nanoparticle as a multi-particle quantum system of charges which combines elements of quantum structures of a polyatomic molecule and a bulk crystal. Considering nanocomposites with quite a low concentration of nanoparticles (fractions of a percent), we didn't take into account interparticle interaction.
\end{abstract}

Keywords: Dielectric Nanocomposite, Quantum Nanostructure, Charge Carriers and Excitons States, Electronic Structure

\section{Introduction}

Optical properties of small particles are being investigated more than 100 years if dating from the Mie's works. However, the intense investigations of these properties have been started in the last decades with intense studies of optical properties of nanocomposites, including heterogeneous liquid-phase nanocomposites (HLPN) based upon semiconductor, metal or structured nanoparticles with both a core and electron shell of different electrical properties [1 - 14]. It is concerned with the fact that nanocomposites based upon semiconductor and dielectric nanoparticles are of special interest for those who develop illumination control means for optical information systems, because of such nanoparticles' great nonlinear-optical response in the near infrared and visible ranges of light.

Nonlinear optical properties of nanocomposites are directly connected with physical processes which occur inside nanoobjects under an influence of a laser radiation, and they depend on electronic structure of nanoparticles. In particular, in metal nanoparticles as well as in dielectric ones with a metal shell, on condition of plasmonic resonance, an increase of effective nonlinearity of the nanocomposite's medium and a decrease in energetic threshold of nonlinear response can occur.

Low nonlinear optical threshold in the ranges of near infrared and visible light can be observed in wide-gap semiconductor nanoparticles as well. For example, the investigation of dependence of the density of energy, passed through a nanoparticle array in transparent dielectric liquid matrix, on the density of incident energy have shown that threshold of nonlinear optical response for the sample based upon $\mathrm{TiO}_{2}$ illuminated with impulses of $\tau$ $=10 \mathrm{~ns}$ on the wavelength of $\lambda=1.06 \mu \mathrm{m}$ is no greater than $0.15 \mathrm{~nJ} / \mathrm{sm}^{2}$, impulses with the wavelength of $0.53 \mu \mathrm{m}$ provided the response less than $0.1 \mathrm{~nJ} / \mathrm{sm}^{2}$. Nonlinear optical properties of nanocomposites based upon dielectric nanoparticles with higher energy-gap width $E_{r}$ were investigated to a less extent. It was concerned, presumably, with the considerations that such energy-gap width could cause the nonlinear optical response of the medium in the ultraviolet range of light. However, recent studies [7, 8] have shown that HLPN based upon dielectric nanoparticles can feature low-threshold nonlinear optical response to an external illumination, impulse and continuous alike, in the near infrared or visible ranges of light.

As it shown in the work [5,6, 12], such media based upon liquid-phase matrix with linear optical properties, being under the influence of nanosecond impulses of near infrared and visible light ranges, display low-threshold (less than 0.5 $\mathrm{nJ} / \mathrm{sm}^{2}$ ) nonlinear optical response. This response is represented in either limitation of passed energy and greater 
medium transparency. The character of this response in these media was tightly bound with a refraction index and an energy gap width of nanoparticles material. The results of the work [7-10] show that the same effects in such media can be witnessed in case of use of continuous laser radiation as well. In both cases modulation of optical properties of liquid heterogeneous medium can be caused by a combination of the next processes: light-induced absorption change, light scattering by nanoparticles, thermal and photoinduced modulation of refraction index.

The light intensity threshold value must depend both upon properties of nanoparticles material and heterogeneous medium matrix material. It gives us an ability to change their nonlinear optical properties by selection of appropriate materials as well as concentration, size and shape of the nanoparticles. The existance of such nonlinear optical properties of nanocomposites indicates that electronic structure of nanoparticles is considerably different from that of a bulk sample. At least these differences are caused, firstly, by formation of areas of allowed energy of charge carriers inside the forbidden band (these areas' structure is connected with a great density of surface defects of the crystal structure and a complex shape of the nanoparticles). Secondly, by the effect of size quantization which causes a change of an exciton energy spectrum and generation of a discrete energy spectrum in a conduction band of free charge carriers, caused by a space limitation of their wave functions localization. Thirdly, electric dipole moments of transitions within such quasizero dimensional objects can be higher than such moments of volume samples [9,10,15-22]. As it was shown during the experimental studies [8,9], a substantial factor which determines nonlinear optical properties of HLPN is a character of dependence of liquid matrix refraction index on the temperature of the matrix medium.

Unfortunately, because of shortage of experimental studies together with even less amount of theoretical studies on nonlinear optical properties of solid and liquid dielectric nanocomposites based upon different dielectric nanoparticles, it is impossible to develop a well-set outlines of physical processes occur within the aforementioned nanocomposites under an influence of an external illumination.

Thus this paper, being based upon the results of our works [1-3, 7-10], deals with experimental and theoretical studies of physical characteristics of nanoparticles responsible for specific optical properties of dielectric nanocomposites. During theoretical description and explanation of experimental data obtained, we considered nanoparticle as a multi-particle quantum system of charges which combines elements of quantum structures of a polyatomic molecule and a bulk crystal. Considering nanocomposites with quite a low concentration of nanoparticles (fractions of a percent), we didn't take into account interparticle interaction.

\section{Peculiarities of Electronic Structure of Dielectric Nanoparticles}

It is discovered nowadays that differences between optical properties of dielectric nanoparticles and that of bulk samples are to a substantial degree bounded to differences between energy spectra of nanoparticle's charge carriers and bulk samples. Mainly, such peculiarities of electronic structure of nanoparticles are caused by their small size and smaller areas of localization of wave functions of their charge carriers. It leads to pronounced quantum-sized effects not only within electronic subsystem of a nanoparticle but within other oscillating subsystems, e.g. phononic ones. Secondly, the peculiarities are caused by nanoparticles' shape which directly affects the energy spectrum of charge carriers and other oscillating subsystems of the nanoparticle [1-3]. The third reason is the existence and a character of nanoparticle's surface defects, inner structure and impurity centers, which influence upon energy spectrum as well as dynamics and relaxation mechanisms of states of charge carriers and other elementary excitations.

The effect of size quantization leads to a radical transformation of quasi-continuous energy spectra and wave functions of charge carriers and elementary excitations of a nanoparticle when its size is decreased. Describing the states, one must take into account a shape and a limited size of a surface which has to satisfy boundary conditions imposed upon wave functions of charge carriers and other elementary excitations. This procedure was carried out carefully enough for the most symmetrical surfaces, e.g. for spherical or parallelepipedic ones. It was discovered that discrete levels energy and energy gap width, regardless of a shape, is inversely proportional to a $v$ power of linear dimension of the nanoparticle when $1 \leq v \leq 2$ [13]. Experimental verification of this dependence has shown that there is only a trend to such dependence which is more pronounced for nanoparticles with size less than 2-3 nm. One of the main reasons of this dependence's disturbance is a deviation of a nanoparticle's shape when compared to the symmetrical one. A modeling of processes of influence of nanoparticle's shape upon an electronic structure of charge carriers and other elementary excitations is a very uncommon issue. However, by using the next approach, we can investigate an influence of nanoparticle's shape upon quantum-size states of its charge carriers [2]. Let us consider an energy spectrum as a functional $\mathrm{E}[\mathrm{s}(\overrightarrow{\mathrm{r}})]$ determined along the space of functions which describe a shape of a nanoparticle's surface $_{s}(\vec{r})$. Presenting this functional as variational series with variations of the surface $\delta s(\vec{r})$. we obtain

$$
\left.E[s(\vec{r})]=E_{0}\left[s_{0}(\vec{r})\right]+\left.\int_{V} \frac{\delta E[s(\vec{r})]}{\delta s(\vec{\xi})}\right|_{S=S_{0}} \delta s(\vec{\xi}) d \vec{\xi}+\left.\frac{1}{2 !} \iint_{V} \frac{\delta^{2} E[s(\vec{r})]}{\delta s\left(\vec{\xi}_{1}\right) \delta s\left(\vec{\xi}_{2}\right)}\right|_{S=S_{0}} \delta s\left(\vec{\xi}_{1}\right) \delta s\left(\vec{\xi}_{2}\right) d \vec{\xi}_{1} d \vec{\xi}_{2}\right)+\ldots
$$


where V - the nanoparticle's volume, $\frac{\delta}{\delta s(\vec{\xi})}$ - variational derivative and $\delta s(\vec{\xi})=s(\vec{\xi})-s_{0}(\vec{\xi})-$ variation of the nanoparticle's surface. Taking into account only the two terms of the variational derivative

$$
\begin{aligned}
& \frac{\delta \mathrm{E}[\mathrm{s}(\overrightarrow{\mathrm{r}})]}{\delta \mathrm{s}(\vec{\xi})}=\frac{\mathrm{dE}[\mathrm{s}(\overrightarrow{\mathrm{r}})]}{\mathrm{ds}(\overrightarrow{\mathrm{r}})} \delta(\overrightarrow{\mathrm{r}}-\vec{\xi}) \text { we have } \\
& \qquad \mathrm{E}[\mathrm{s}(\overrightarrow{\mathrm{r}})] \approx \mathrm{E}\left[\mathrm{s}_{0}(\overrightarrow{\mathrm{r}})\right]+\left.\frac{\mathrm{dE}[\mathrm{s}(\overrightarrow{\mathrm{r}})]}{\mathrm{ds}(\overrightarrow{\mathrm{r}})}\right|_{\mathrm{s}=\mathrm{s}_{0}} \delta \mathrm{s}(\overrightarrow{\mathrm{r}}) .
\end{aligned}
$$

It is convenient to choose a $s_{0}(\vec{r})$ surface of a simple and the most symmetrical form in order to obtain a wellknown energy spectrum of charge carriers. Let us assume that an undisturbed surface is a sphere $-\mathrm{s}_{0}(\overrightarrow{\mathrm{r}})=\mathrm{R}_{0}^{2}$ and proceed into a spherical coordinate system (SCS). In this case the distance between the centre of the sphere and a point upon its surface $\mathrm{R}(\theta, \phi)$ will satisfy the condition $\mathrm{R}_{0}^{2}(\theta, \varphi)=\mathrm{R}_{0}^{2}$. Within our spherical coordinate system a variation of the surface is determined by the next expression

$$
\delta \mathrm{s}(\mathrm{R}, \theta, \phi)=\mathrm{s}(\mathrm{R}, \theta, \phi)-\mathrm{s}_{0}(\mathrm{R}, \theta, \phi)=\delta \mathrm{R}^{2}(\theta, \phi)=\mathrm{R}^{2}(\theta, \phi)-\mathrm{R}_{0}^{2}
$$

where $R^{2}(\theta, \phi)$ - the square distance between the centre of the sphere and a point upon disturbed surface $s(R, \theta, \phi)$. It is known that energy of a free charge carrier inside the infinitely deep spherical hole with radius $R_{0}$ is determined by the expression $E_{n, 1}\left(R_{0}\right)=\frac{\alpha_{n, 1}^{2} \hbar^{2}}{2 R_{0}^{2} m}$, where $\alpha_{n, 1}$ - roots of the Bessel function, $\mathrm{m}$ - the mass of a charge carrier. Introducing the designation $\mathrm{E}_{\mathrm{n}, \mathrm{l}}\left(\mathrm{R}_{0}\right)=\mathrm{E}_{\mathrm{n}, \mathrm{l}}^{0}$ from Eq. (1) we obtain -

$$
\mathrm{E}_{\mathrm{nl}}(\theta)=\mathrm{E}_{\mathrm{nl}}^{0}\left(1-\frac{\delta \mathrm{R}^{2}(\theta, \varphi)}{\mathrm{R}_{0}^{2}}\right)
$$

The angle $\theta$ in Eq. (2) should be considered as an angle between a vector of the particle's angular momentum and a chosen spatial direction. There is a preferential spatial direction which corresponds to $\mathrm{OZ}$ axis. When the particle's plane of motion makes an angle $\theta$ with $\mathrm{OZ}$ axis then $\cos ^{2} \theta=\frac{\mathrm{m}^{2}}{1(1+1)}$, where $\ell$ and $\mathrm{m}$ - orbital and magnetic quantum numbers correspondingly. Quantum states density is equal to, $N(E)=J_{g} \frac{4 \pi \rho^{2}}{(2 \pi \hbar)^{3}} \frac{d P}{d E}$ where $J_{g}$ - amount of states that are not associated with spatial movement of the particle, $\mathrm{P}$ - impulse of the particle. In case of electrons or electron holes $\mathrm{P}=\sqrt{2 \mathrm{mE}}$ $\mathrm{J}_{\mathrm{g}}=2 \quad \mathrm{~N}(\mathrm{E})=2 \frac{4 \pi \rho^{2}}{(2 \pi \hbar)^{2}} \frac{\mathrm{dP}}{\mathrm{dE}}=\frac{\sqrt{2} \mathrm{~m}^{\frac{3}{2}}}{\pi^{2} \hbar^{3}} \mathrm{~V} \sqrt{\mathrm{E}}, \mathrm{V}$ - nanoparticle's volume, $\mathrm{m}$ - effective mass of electron or electron hole. Density of states is a functional defined in the space of functions which describe a geometrical shape of a nanoparticle. Thus we can consider $\mathrm{N}(\mathrm{E})$ as
$\mathrm{N}(\mathrm{E})=\left.\mathrm{N}(\mathrm{E})\right|_{\mathrm{s}(\overrightarrow{\mathrm{r}})=\mathrm{s}_{0}}+\left.\int_{\mathrm{V}} \frac{\delta \mathrm{N}(\mathrm{E})}{\delta \mathrm{s}(\vec{\xi})}\right|_{\mathrm{s}_{0}} \delta \mathrm{s}(\vec{\xi}) \mathrm{d} \vec{\xi}+\ldots$ and we can observe that for a small disturbance of the surface $\delta s(\vec{r})$ the change of the quantum states density is determined as $\left.\Delta \mathrm{N}(\mathrm{E}) \approx \int_{\mathrm{V}} \frac{\delta \mathrm{N}(\mathrm{E})}{\delta \mathrm{s}(\vec{\xi})}\right|_{\mathrm{s}_{0}} \delta \mathrm{s}(\vec{\xi}) \mathrm{d} \vec{\xi}$. For quantum-size states we obtain the next expression

$$
\Delta \mathrm{N}(\mathrm{E})=\left.\frac{\mathrm{m}^{312} \mathrm{v}}{\sqrt{2} \pi^{2} \hbar^{3} \sqrt{\mathrm{E}\left[\mathrm{s}_{0}(\overrightarrow{\mathrm{r}})\right]}} \frac{\delta \mathrm{E}[\mathrm{s}(\overrightarrow{\mathrm{r}})]}{\delta \mathrm{s}(\vec{\xi})}\right|_{\mathrm{s}_{0}} \delta \mathrm{s}(\vec{\xi})=\frac{\mathrm{m}^{312} \mathrm{v}}{\sqrt{2} \pi^{2} \hbar^{3}} \frac{\Delta \mathrm{E}[\delta \mathrm{s}(\overrightarrow{\mathrm{r}})]}{\sqrt{\mathrm{E}\left[\mathrm{s}_{0}(\overrightarrow{\mathrm{r}})\right]}},
$$

where $\Delta \mathrm{E}[\delta \mathrm{s}(\overrightarrow{\mathrm{r}})]=\mathrm{E}[\mathrm{s}(\overrightarrow{\mathrm{r}})]-\mathrm{E}\left[\mathrm{s}_{0}(\overrightarrow{\mathrm{r}})\right]$.

Let us consider several examples of the approach presented. In a general case we represent an axialsymmetrical surface of a nanoparticle with the next equation $\mathrm{R}^{2}(\theta)=\mathrm{R}_{0}^{2}\left[1+\mathrm{p}^{2} \cos ^{\gamma}(\mathrm{k} \theta)\right]$. Let $\mathrm{p}^{2}=0,2, \gamma=2, \mathrm{k}=3$. This nanoparticle has the shape shown in Fig. 1. Relationships between energy spectrum and the density of quantum-size states of this nanoparticle and the nanoparticle of a spherical shape with radius $\mathrm{R}_{0}$ are equal to

$$
\frac{\mathrm{E}\left[\mathrm{R}^{2}\right]}{\mathrm{E}\left[\mathrm{R}_{0}^{2}\right]}=\left\{1-0,2\left[\frac{4 \mathrm{~m}^{2}}{\ell(\ell+1)}-3\left(\frac{\mathrm{m}^{2}}{\ell(\ell+1)}\right)^{\frac{1}{2}}\right]\right\}^{2} \frac{\mathrm{N}\left[\mathrm{R}_{0}^{2}\right]}{\mathrm{N}\left[\mathrm{R}^{2}\right]}=\left(\frac{1}{2}+\frac{\mathrm{E}\left[\mathrm{R}^{2}(\theta)\right]}{2 \mathrm{E}\left[\mathrm{R}_{0}^{2}\right]}\right)
$$

The plots displaying such these relationships are shown in Fig.(1).

When $\gamma=3, \mathrm{k}=2$ the abovementioned relationships have the next form: $\frac{\mathrm{E}\left[\mathrm{R}^{2}\right]}{\mathrm{E}\left[\mathrm{R}_{0}^{2}\right]}=1-0,2\left[\frac{2 \mathrm{~m}^{2}}{\ell(\ell+1)}-1\right]^{3}$, $\frac{\mathrm{N}\left[\mathrm{R}_{0}^{2}\right]}{\mathrm{N}\left[\mathrm{R}^{2}\right]}=\left(1-0,1\left[\frac{2 \mathrm{~m}^{2}}{\ell(\ell+1)}-1\right]^{3}\right)$. The corresponding plots are shown in Fig. (2).
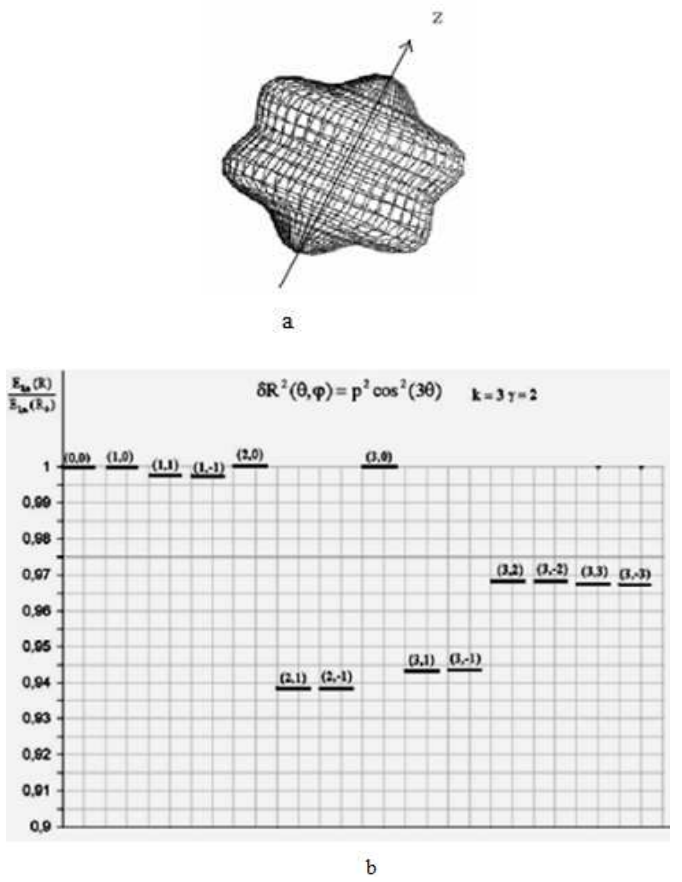


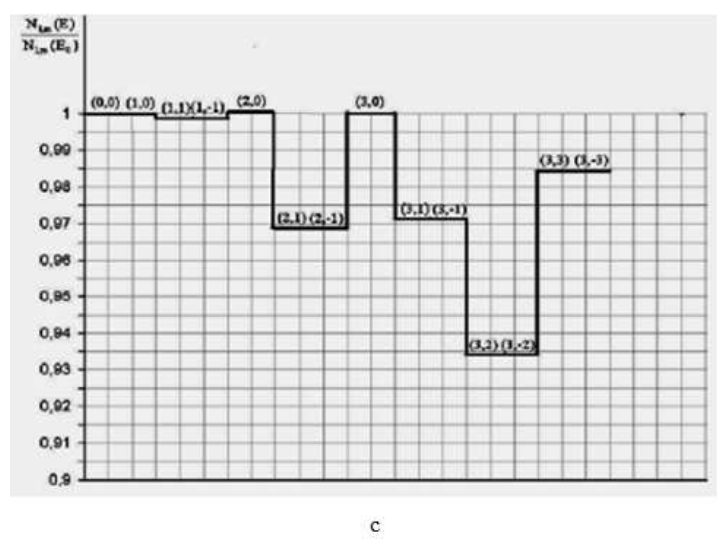

Fig. 1. (a) - 3D-model of a nanoparticle; (b) - Relationship between energy spectra and $(c)$ - quantum-size states

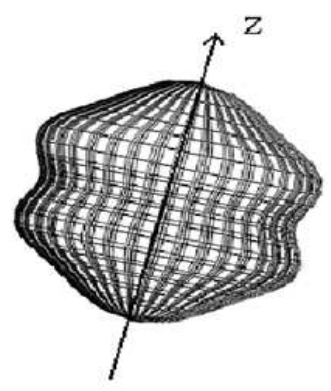

a

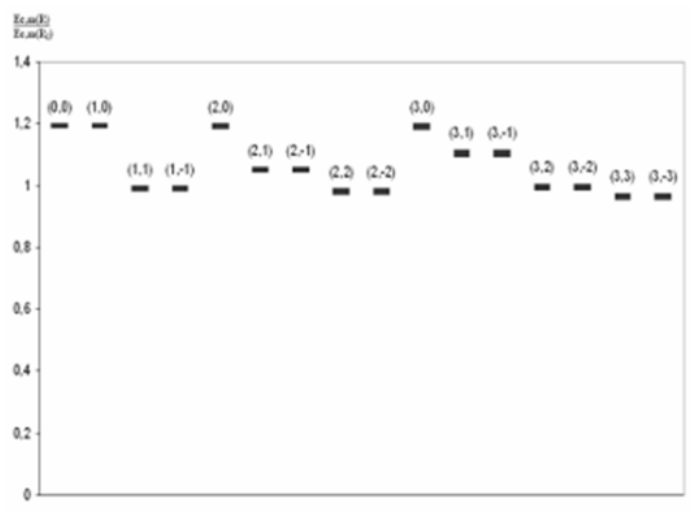

b

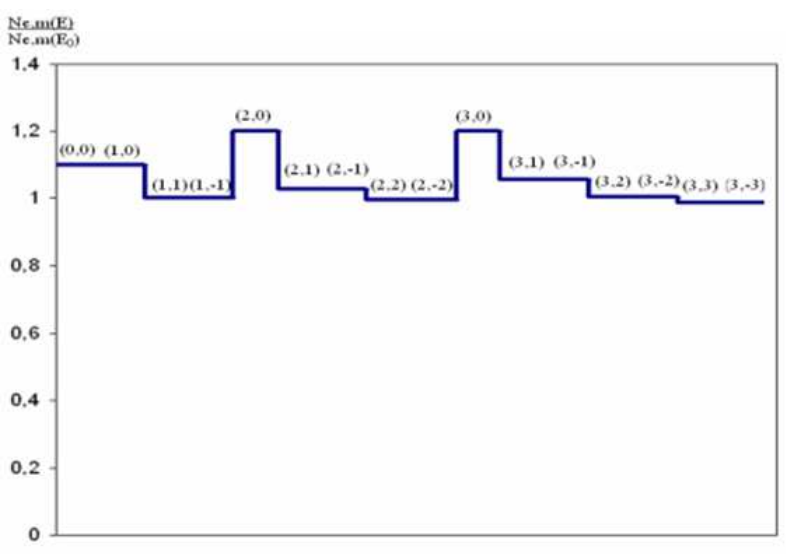

c

Fig. 2. (a) - 3D-model of a nanoparticle; (b) - Relationship between energy spectra and (c) quantum-size states.
The examples demonstrate that nanoparticle's shape deviation from the spherical one lessens the degree of magnetic quantum number degeneracy of charge carriers energy. In addition, this deviation leads to a formation of allowed energy minizones which lie above (Fig. 2) as well as below (Fig. 1) non-split levels of a spherical nanoparticle. It causes a corresponding change of quantumstates density which can be greater (Fig.1) or lesser (Fig.2) than a base level.

Previously we considered single-particle states of charge carriers, but formation of optical properties of dielectric and semiconductor nanostructures depends mostly upon double-particle states also known as exciton states. A contribution of exciton states into optical properties is substantial if their Bohr radius is comparable to nanoparticle's size (or less than the size - the weak confinement regime). In contrast to the single-particle states, the accurate description of exciton states is impossible even for spherical nanoparticles. Use of approximate approaches which utilize a model of a nanoparticle represented as a deep potential hole of a spherical shape, and modeling the influence of a boundary upon charge carriers by a quantum hole potential allowed us to make some overall conclusions on energy spectrum of exciton within a nanoparticle $[3,4]$.

First of all, every size of a nanoparticle is associated with a finite amount of levels with negative exciton binding energy; this amount is decreasing when an exciton is lessening. There is a critical nanoparticle's size such that these levels disappear. Secondly, the orbital quantum state degeneracy of a relative motion, peculiar to Coulomb motion in infinite medium is removed.

We can investigate an influence of nanoparticles' shape and size upon exciton energy spectrum more carefully by using an exactly solvable model of a nanoparticle represented as a system of two charge carriers - electron and electron hole, which exist inside an infinitely deep potential hole limited by paraboloid of revolution and sizes of real nanoparticles $[9,10]$. We estimate exciton energy spectrum taking into account only Coulomb interaction between electron and electron hole as well as quantum size effect in the effective mass approach. This assumption is reasonable due to comparatively small sizes of nanoparticles. Exciton's wave function $\Psi(\eta, \xi, \varphi)$ in a parabolic coordinate system with the centre in the centre of gravity of electron-hole pair satisfies the next equation [11]

$$
\frac{4}{\xi+\eta}\left[\frac{\partial}{\partial \xi}\left(\xi \frac{\partial \Psi}{\partial \xi}\right)+\frac{\partial}{\partial \eta}\left(\eta \frac{\partial \Psi}{\partial \eta}\right)\right]+\frac{1}{\xi \eta} \frac{\partial^{2} \Psi}{\partial \varphi^{2}}+2\left(E+\frac{2}{\xi+\eta}\right)=0 .
$$

Eq. (4) utilizes relative units where the Plank constant and a charge are equal to 1 , masses of electron $m_{e}$, electron hole $\mathrm{m}_{\mathrm{h}}$ and exciton $\mu=\frac{m_{e} m_{h}}{m_{e}+m_{h}}$ are chosen in accordance with the effective in accordance with the effective mass approach; as a length unit Bohr radius of the exciton. . Parabolic coordinates are 
connected with Cartesian ones as it follows $x=\sqrt{\xi \eta} \cos \phi \quad, y=\sqrt{\xi \eta} \sin \phi \quad, z=\frac{1}{2}(\xi-\eta)$.

Paraboloid of revolution around the $\mathrm{OZ}$ axis in parabolic coordinates system is represented with the equation $\eta=\eta_{0}$ (smaller values of $\eta_{0}$ correspond to a narrower paraboloid); a surface $\mathrm{Z}=\mathrm{Z}_{0}$ which is a base of nanoparticle is represented with the equation $\xi=2 z_{0}+\eta$. Coordinates of nanoparticle's surface points satisfy the equations $\eta=\eta_{0}$ and $\xi=2 z_{0}+\eta$. The corresponding boundary condition imposed upon the wave function on the surface of the potential hole has the next form $\Psi\left(\eta_{0}, \xi=2 z_{0}+\eta, \varphi\right)=0$. A solution of this equation which is finite in the centre of coordinate system and zero-equal on the infinity is expressed by using degenerated hypergeometric functions $F(\alpha,|m|+1, \rho)$. This solution has the next form

$$
\begin{gathered}
\Psi(\eta, \xi, \varphi)=\mathrm{C} \frac{1}{\mathrm{n}^{2}|\mathrm{~m}| !} \sqrt{\frac{(\alpha+|\mathrm{m}|) !(\beta+|\mathrm{m}|) !}{\alpha ! \beta !}} \times \\
\times F\left(-\alpha,|m|+1, \frac{\xi}{n}\right)_{F}\left(-\beta,|m|+1, \frac{\eta}{n}\right) \exp \left(-\frac{\xi}{2 n}-\frac{\eta}{2 n}+i m \varphi\right)\left(\frac{\xi \eta}{n^{2}}\right)^{|m|}
\end{gathered}
$$

The $\mathrm{n}$ number defines exciton's binding energy levels $\mathrm{E}_{\mathrm{n}}=-\frac{\mu \mathrm{e}^{4}}{2 \hbar^{2} \varepsilon_{2}^{2} \mathrm{n}^{2}}=-\frac{\mu}{\mathrm{m}_{\mathrm{o}} \varepsilon_{2}^{2} \mathrm{n}^{2}} 13,6 \mathrm{eV}$. There is a ground quantum state with $\beta=1, \alpha=0$ and $\mathrm{n}(|\mathrm{m}|+1)=\eta_{0}$ which satisfies the boundary condition upon the side surface of the paraboloid of revolution. In this case $\mathrm{n}=\frac{|\mathrm{m}|+1}{2} \pm \sqrt{\frac{(|\mathrm{m}|+1)^{2}}{4}+\frac{\eta_{0}}{|\mathrm{~m}|+1}}$ and every value of quantum number $\mathrm{m}$ is associated with two levels. The value of $\mathrm{m}=0$ corresponds to an exciton which has a motion plane parallel to OZ. The effective masses of electron and electron hole in, e.g. Al2O3 are equal to $0.4 \mathrm{~m}_{0}$ and $6.2 \mathrm{~m}_{0}$. Given these masses within the nanoparticle of $40 \mathrm{~nm}$ height and $40 \mathrm{~nm}$ width of a bottom the value of $\eta_{0}$ is equal to 13 a.u. and the binding energies of the exciton's ground state are equal to $0.07 \mathrm{eV}$ and $-0.04 \mathrm{eV}$ correspondingly. Assuming the kinetic energy of exciton's motion to be negligibly small, we come to the conclusion that energy levels of the exciton lie inside the forbidden band at a ranges of $0.07 \mathrm{eV}$ and $0.04 \mathrm{eV}$ from the bottom of conduction band.

The second duplet of levels corresponding to $\mathrm{m}=1$ lies at range $0.05 \mathrm{eV}$ and $0.21 \mathrm{eV}$ from the conduction band to the direction of forbidden band. These values for $\mathrm{m}=2,3,4$ are equal to $0.03 \mathrm{eV}$ and $0.58 \mathrm{eV}, 0.04 \mathrm{eV}$ and $1.36 \mathrm{eV}, 0.02$ and $4.06 \mathrm{eV}$ correspondingly. One can observe the effect of geometrical amplification of electronhole interaction in the spectrum of the abovementioned lines. The boundary condition upon the surface $\xi=2 z_{0}+\eta$ is assosiateld with excited state with $\mathrm{n}=\frac{|\mathrm{m}|+1}{2} \pm \sqrt{\frac{(|\mathrm{m}|+1)^{2}}{4}+\frac{2 \mathrm{z}_{0}+\eta}{|\mathrm{m}|+1}}$. One can witness in this state a dependence of energy levels on coordinate $\eta$ hich varies in the range from 0 to $\eta_{0}$ The dependence demonstrates an influence of a surface shape of a potential hole upon exciton's energy spectrum. Generally, the exciton's energy spectrum remains constant. It consists of two areas: the first one is virtually continuous and it lies next to the bottom of the conduction band, the second one is discrete and it lies in the deep of the forbidden band.

In the effective mass approach the wave function $\Psi(\eta, \xi, \varphi)$ of a free motion of the nanoparticle within the potential hole in parabolic system of coordinates satisfies the Eq. (4) considered without the Coulomb's term. This equation's solution, which is finite in the centre of the coordinate system, is known and is expressed by using Bessel functions $\Psi(\eta, \xi, \varphi)=\mathrm{CJ}_{\mathrm{m} / 2}\left(\frac{\mathrm{k}}{2} \eta\right) \mathrm{J}_{\mathrm{m} / 2}\left(\frac{\mathrm{k}}{2} \xi\right) \exp (\mathrm{m} \varphi)$. The spectrum of free particles is $\mathrm{E}_{\mathrm{n}, \mathrm{m}}=\frac{2 \hbar^{2}}{\mu_{\mathrm{e}, \mathrm{h}} \mathrm{m}_{0}} \alpha_{\mathrm{n}, \mathrm{m}}^{2}\left[\frac{1}{\eta_{0}^{2}}+\frac{1}{\left(2 \mathrm{z}_{0}+\eta\right)^{2}}\right]$, $\mu_{\mathrm{e}, \mathrm{h}}$ - relative mass of an electron or an hole.

Energy spectrum of free charge carriers like energy spectrum of an exciton is dependent of $\eta$.

Thus, taking into account natural and thermal broadening of levels, we can say that energy spectrum of the exciton is formed as a virtually continuous band with a width of $0.1 \mathrm{eV}$ approx., adjacent from the bottom to the conduction band, and broadened discrete levels which lie within the forbidden band at the range about $0.2 \mathrm{eV}$ from the bottom of conduction band. Energy spectrum of charge carriers in the conduction band can have discrete levels as well.

The above results may be summed up as follows: we can build up a model of a diagram of energy levels of singleand double-particles charge carriers states in dielectric nanoparticles (Fig. 3) by using $\mathrm{Al}_{2} \mathrm{O}_{3}$ nanoparticles as an example. This particle is of a complex shape and its sizes are equal to 40-50 $\mathrm{nm}$ approx. (Fig. 4).
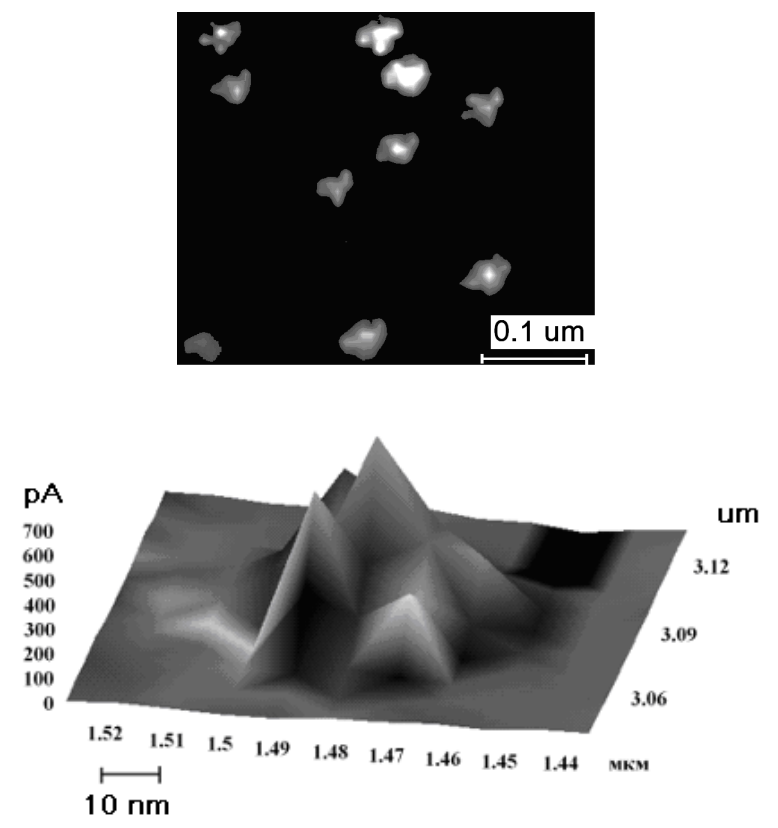

Fig. 3. Images of Al2O3 nanoparticles obtained by using SAFM. 


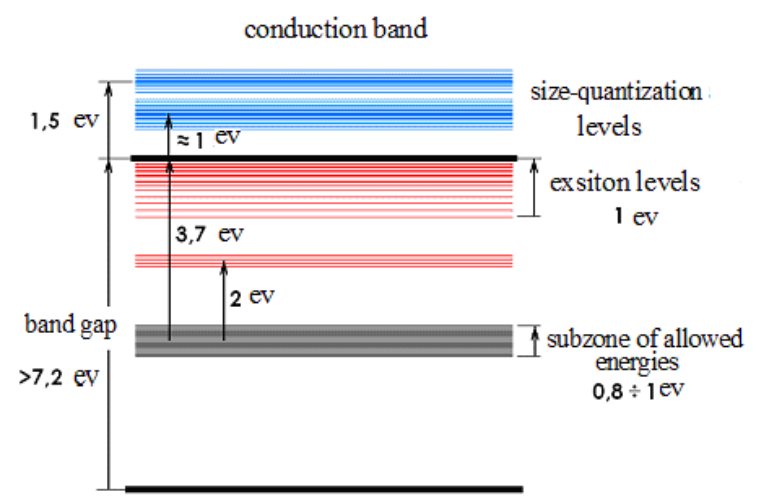

Fig. 4. The diagram of energy levels of single-and double-particles states of charge carriers in dielectric nanoparticles.

Unlike volume samples, the nanoparticles charge carriers can have an energy spectrum with broadened quantum size levels, broad band of exciton states and a subzone of allowed energies, lying within forbidden band, caused by surface and internal defects of nanoparticle's material, impurity centers etc., It should be noted that electronic structure of nanoparticle within a matrix depends to a great extent on relationship of dielectric permittivities $\frac{\varepsilon_{1}}{\varepsilon_{2}}$ of materials of matrix and nanoparticles [15].When $\frac{\varepsilon_{1}}{\varepsilon_{2}}>1$ polarization interaction leads to positive charges (holes) attraction to the inner surface of a nanoparticle and to exciton destruction. Internal surface states are established and their contribution to the subzone of allowed energies causes its drift towards valence band. When $\frac{\varepsilon_{1}}{\varepsilon_{2}}<1$ polarization interaction leads to a repulsion of charges from the nanoparticle's surface towards the inside so the exciton states are formed [15]. However, if nanoparticle's sizes are small, we have $\mathrm{a}<\mathrm{a}_{\mathrm{c}}=6\left|\frac{\varepsilon_{1}+\varepsilon_{2}}{\varepsilon_{1}-\varepsilon_{2}}\right| \mathrm{a}_{\mathrm{b}}$, where $\mathrm{a}_{\mathrm{b}}-$ Bohr radius of charge carrier in the nanoparticle. Otherwise the energy spectrum will be defined by quantum-size states.

\subsection{Absorption and Scattering Cross-Sections of Dielectric Nanoparticles and Transmission Spectrum of HLPN}

One of the most important and informative optical characteristics of electronic structure and physical mechanisms responsible for optical properties of HLPN in near infrared and ultraviolet light are cross-sections of absorption $\sigma_{\mathrm{a}}(\omega, \mathrm{a})$ and scattering $\sigma_{\mathrm{s}}(\omega, \mathrm{a})$ of light by nanoparticles as well as light transmission spectrum of HLPN - T $(\omega, \mathrm{a}, \mathrm{N})$. Cross-sections $\sigma_{\mathrm{s}}(\omega, \mathrm{a})$ and $\sigma_{\mathrm{a}}(\omega, \mathrm{a})$ and their dependence upon nanoparticle's shape and light frequency $\boldsymbol{\omega}$ can be defined in terms of polarizability of nanoparticle's volume unit $A(\omega, a)$ with the next expressions .

$$
\sigma_{\mathrm{a}}(\omega, \mathrm{a})=\frac{4 \pi \omega}{\mathrm{c}} \mathrm{V} \operatorname{Im} \mathrm{A}(\omega, \mathrm{a}), \mathrm{d} \sigma_{\mathrm{s}}(\omega, \mathrm{a})=\frac{\omega^{4}}{\mathrm{c}^{4}} \mathrm{~V}^{2}|\mathrm{~A}(\omega, \mathrm{a})|^{2} \sin ^{2} \theta \mathrm{d} \Omega,
$$

where $\theta$ - angle between a direction of a scattering vector and electric field vector, $\mathrm{c}-$ the speed of light in vacuum. Within the framework of dipole approach a polarizabilty of nanoparticle's volume unit is defined by $D_{i j}(a)$ elements of the matrix of electric dipole moment of transition between $|i\rangle$ and $|\mathbf{j}\rangle$ states -

$$
\mathrm{A}(\omega, \mathrm{a})=\frac{2}{\mathrm{~V} \hbar}\left[\sum_{\mathrm{i}, \mathrm{j}} \frac{\left|\mathrm{D}_{\mathrm{ij}}(\mathrm{a})\right|^{2}\left(\omega_{\mathrm{j}}^{2}-\omega^{2}\right)}{\left(\omega_{\mathrm{j}}^{2}\right)^{2}+\omega^{2} \Gamma_{\mathrm{j}}^{2}} \omega_{\mathrm{j}}+\mathrm{i} \sum_{\mathrm{i}, \mathrm{j}} \frac{\left|\mathrm{D}_{\mathrm{ij}}(\mathrm{a})\right|^{2} \Gamma_{\mathrm{j}}}{\left(\omega^{2}-\omega_{\mathrm{j}}^{2}\right)^{2}+\omega^{2} \Gamma_{j}^{2}} \omega \omega_{\mathrm{j}}\right]
$$

where summation is carried out over all dipole moments excited by a laser radiation, $\omega_{j}$ - transition frequency, $\Gamma_{j}^{-}$ width of excited level, $\hbar$ - Plank's constant. It follows from the Eq. (5) and (6) that expressions for absorption cross-section and scattering cross-section of nanoparticle should be written as

$$
\sigma_{\mathrm{a}}(\omega, \mathrm{a})=\frac{8 \pi}{\mathrm{c} \hbar} \sum_{\mathrm{i}, \mathrm{j}} \frac{\left|\mathrm{D}_{\mathrm{ij}}(\mathrm{a})\right|^{2} \omega_{\mathrm{j}} \Gamma_{\mathrm{j}}}{\left(\omega^{2}-\omega_{\mathrm{j}}^{2}\right)^{2}+\omega^{2} \Gamma_{\mathrm{j}}^{2}} \omega^{2}
$$
[15].

$$
\sigma_{\mathrm{s}}(\omega, \mathrm{a})=\frac{4 \mathrm{P} \omega^{4}}{\mathrm{c}^{4} \hbar^{2}} \sum_{\mathrm{i}, \mathrm{j}} \sum_{\mathrm{i}, \mathrm{k}}\left\{\frac{\left|\mathrm{D}_{\mathrm{ij}}(\mathrm{a})\right|_{\mid}^{2}\left|\mathrm{D}_{\mathrm{ik}}(\mathrm{a})\right|^{2} \omega_{\mathrm{j}} \omega_{\mathrm{k}}\left[\left(\omega_{\mathrm{j}}^{2}-\omega^{2}\right)\left(\omega_{\mathrm{k}}^{2}-\omega^{2}\right)+\Gamma_{\mathrm{j}} \Gamma_{\mathrm{k}} \omega^{2}\right]}{\left[\left(\omega_{\mathrm{k}}^{2}-\omega^{2}\right)^{2}+\omega^{2} \Gamma_{\mathrm{k}}^{2}\left[\left(\omega_{\mathrm{j}}^{2}-\omega^{2}\right)^{2}+\omega^{2} \Gamma_{j}^{2}\right]\right.}\right\},
$$

where $P=\oint_{4 \pi} \sin ^{2} \theta d \Omega$. Dependence of the cross-sections on nanoparticle's size can be found knowing a relation between values of $D_{i j}(a), \omega_{j}, \Gamma_{j}$ and nanoparticle's size. The value of matrix element of a dipole moment of a state $|i\rangle$ to a state $|j\rangle$ transition is $D_{i j}(a)=\Lambda\left(\varepsilon_{1}, \varepsilon_{2}\right) e\langle j|\vec{r} \gamma| i\rangle$, where $\overrightarrow{\mathbf{r}}$ - radius-vector of a charge, $\vec{\gamma}$ - vector of polarization direction of the electromagnetic wave which induced the abovementioned transition. The factor $\Lambda\left(\varepsilon_{1}, \varepsilon_{2}\right)$ is responsible for nanoparticle's shape as well as for dielectric permittivities of nanoparticle and medium surrounding this nanoparticle. The theorem on a mean value of definite integral as well as from wave function normalization imply that $\mathrm{D}_{\mathrm{ij}}(\mathrm{a})=\Lambda\left(\varepsilon_{1}, \varepsilon_{2}\right) e \Psi_{[j]}^{*}\left(\overrightarrow{\mathrm{r}}_{1}\right) \Psi_{[\mathrm{i}]}\left(\overrightarrow{\mathrm{r}}_{1}\right) \int_{\mathrm{V}} \overrightarrow{\mathrm{r}} \overrightarrow{\mathrm{d}} \overrightarrow{\mathrm{r}}=\mathrm{d}_{\mathrm{ij}} \mathrm{a}$, where $\overrightarrow{\mathrm{r}}_{1}$ - radiusvector of a point inside the nanoparticle, value of $\mathrm{d}_{\mathrm{ij}}$ is directly related to a shape of nanoparticle. The linear dependence of $D_{i j}(a)$ upon a shape of nanoparticle is valid for any a smaller than $a_{c}$. Reaching this limit we must take into account polarization interaction between charge carriers and charge induced at the nanoparticle-medium 
interface. On condition that nanoparticle's size exceeds $a_{c}$, the $D_{i j}(a)$ is defined by a size of localization area of dipole transitions states. In case of a transition to an area with continuous spectrum a matrix transition element will be proportional to an area of charge carrier's delocalization $a$ approx., i.e. as in case of bound states. Thus, considering dipole moments of transition towards quantum-size states $\mathrm{D}_{\mathrm{ij}}^{\mathrm{q}}(\mathrm{a})$, exciton states $\mathrm{D}_{\mathrm{ij}}^{\mathrm{ex}}(\mathrm{a})$ and continuous spectrum states $\mathrm{D}_{\mathrm{ij}}^{\mathrm{c}}(\mathrm{a})$, we can write: $\mathrm{D}_{\mathrm{ij}}^{\mathrm{q}}(\mathrm{a})=\mathrm{d}_{\mathrm{ij}}^{\mathrm{q}} \mathrm{a}, D_{i j}^{e x}(a)=d_{i j}^{e x} a$ and $D_{\mathrm{ij}}^{\mathrm{c}}(\mathrm{a})=\mathrm{d}_{\mathrm{ij}}^{\mathrm{c}} \mathrm{a}$. Frequencies of transitions to quantum size states $\omega_{j}=\frac{E_{j}}{\hbar}=\frac{\hbar \phi_{j}^{2}}{2 m_{e, h} a^{2}} \chi=\frac{c_{j}}{a^{2}}$, where $\phi_{j}^{2}$ - roots of Schrodinger equation for free electrons (electron holes)

$$
\sigma_{\mathrm{s}}^{q}(\omega, \mathrm{a})=\frac{4 \mathrm{P} \omega^{4}}{\mathrm{c}^{4} \hbar^{2}} \sum_{\mathrm{i}, \mathrm{j}} \sum_{\mathrm{i}, \mathrm{k}}\left\{\frac{\mathrm{d}_{\mathrm{ijj}}^{2} \mathrm{~d}_{\mathrm{ik}}^{2} \mathrm{c}_{\mathrm{j}} \mathrm{c}_{\mathrm{k}}\left[\left(\mathrm{c}_{\mathrm{j}}^{2}-\mathrm{a}^{4} \omega^{2}\right)\left(\mathrm{c}_{\mathrm{k}}^{2}-\mathrm{a}^{4} \omega^{2}\right)+\mathrm{a}^{8} \Gamma_{\mathrm{j}} \Gamma_{\mathrm{k}} \omega^{2}\right]}{\left[\left(\mathrm{c}_{\mathrm{k}}^{2}-\mathrm{a}^{4} \omega^{2}\right)^{2}+\mathrm{a}^{8} \omega^{2} \Gamma_{\mathrm{k}}^{2}\right]\left[\left(\mathrm{c}_{\mathrm{j}}^{2}-\mathrm{a}^{4} \omega^{2}\right)^{2}+\mathrm{a}^{8} \omega^{2} \Gamma_{\mathrm{j}}^{2}\right]} \mathrm{a}^{8}\right\}
$$

towards exciton states

$$
\begin{gathered}
\sigma_{\mathrm{a}}^{\mathrm{ex}}(\omega, \mathrm{a})=\frac{8 \pi}{\mathrm{c} \hbar} \sum_{\mathrm{i}, \mathrm{j}} \frac{\mathrm{d}_{\mathrm{ij}}^{\mathrm{ex}} \mathrm{a}^{2} \omega_{\mathrm{j}} \Gamma_{\mathrm{j}}}{\left(\omega^{2}-\omega_{\mathrm{j}}^{2}\right)^{2}+\omega^{2} \Gamma_{j}^{2}} \omega^{2} \\
\sigma_{\mathrm{s}}^{\text {ex }}(\omega, \mathrm{a})=\frac{4 \mathrm{P} \omega^{4}}{\mathrm{c}^{4} \hbar^{2}} \sum_{\mathrm{i}, \mathrm{i}} \sum_{\mathrm{i}, \mathrm{k}}\left\{\frac{\mathrm{d}_{\mathrm{ij}}^{2} \mathrm{~d}_{\mathrm{i}}^{2} \omega_{\mathrm{j}} \omega_{\mathrm{k}}\left[\left(\omega_{\mathrm{j}}^{2}-\omega^{2}\right)\left(\omega_{\mathrm{k}}^{2}-\omega^{2}\right)+\omega^{2} \Gamma_{\mathrm{j}} \Gamma_{\mathrm{k}}\right]}{\left[\left(\omega_{\mathrm{k}}^{2}-\omega^{2}\right)^{2}+\omega^{2} \Gamma_{\mathrm{k}}^{2}\right]\left[\left(\omega_{\mathrm{j}}^{2}-\omega^{2}\right)^{2}+\omega^{2} \Gamma_{\mathrm{j}}^{2}\right]} \mathrm{a}^{4}\right\}
\end{gathered}
$$

For transitions towards continuous spectrum with $\omega_{j}=\omega$

$$
\sigma_{\mathrm{a}}^{\mathrm{c}}(\omega, \mathrm{a})=\frac{8 \pi}{\mathrm{c} \hbar} \sum_{\mathrm{i}, \mathrm{j}} \frac{\mathrm{d}_{\mathrm{ij}}^{\mathrm{c} 2} \mathrm{a} \omega}{\Gamma_{\mathrm{j}}}, \sigma_{\mathrm{s}}^{\mathrm{c}}(\omega, \mathrm{a})=\frac{4 \mathrm{P} \omega^{4}}{\mathrm{c}^{4} \hbar^{2}} \sum_{\mathrm{i}, \mathrm{j}} \sum_{\mathrm{i}, \mathrm{k}}\left\{\frac{\mathrm{d}_{\mathrm{id}}^{2} \mathrm{~d}_{\mathrm{ik}}^{2}}{\Gamma_{\mathrm{j}} \Gamma_{\mathrm{k}}} \mathrm{a}^{4}\right\} .
$$

The expression for transmission spectrum $\mathrm{T}$ of a flat layer with a thickness of $h(h>>\lambda)$ of a nanocomposite during a normal light incidence is

$$
T(\omega, a, N)=\frac{\left(1-R^{2}\right)^{2} \exp (-\alpha h)}{1-R^{2} \exp (-2 \alpha h)},
$$

where $\mathrm{R}$ - coefficient of light reflection from the layer's edge which is, as a rule, much smaller than 1 on experimental conditions. Extinction coefficient $\alpha$ is expressed by abovementioned cross-sections of absorption and scattering, a number $N$ of nanoparticles per volume unit - $\quad \alpha=\mathrm{N}\left[\sigma_{\mathrm{a}}(\omega, \mathrm{a})+\sigma_{\mathrm{s}}(\omega, \mathrm{a})\right]+\alpha_{\mathrm{m}}(\omega) \quad$ and coefficient of laser light attenuation by matrix medium $\alpha_{\mathrm{m}}(\omega)$. The ratio of cross-sections of absorption to scattering, on condition that the width of the $j$-th excited level is only slightly dependent of $j$, is $\frac{\sigma_{\mathrm{s}}(\omega, \mathrm{a})}{\sigma_{\mathrm{a}}(\omega, \mathrm{a})}=\frac{\mathrm{P} \omega^{3}}{2 \pi \mathrm{c}^{3} \hbar \Gamma} \sum_{\mathrm{i}, \mathrm{j}}\left|\mathrm{D}_{\mathrm{ij}}(\mathrm{a})\right|^{2} \sim \omega^{3} \mathrm{a}^{2}$. For nanoparticles with size within range from 10 to $100 \mathrm{~nm}$ in the frequency range from $10^{13}$ to $10^{16} \mathrm{~Hz}$ and when $\Gamma=10^{9} \mathrm{~Hz}$, the ratio of inside the potential hole, defined from the conditions upon the boundaries of the hole, $\mathrm{m}_{\mathrm{e}}$ and $\mathrm{m}_{\mathrm{h}}$ - effective masses of an electron and a hole correspondingly, $\chi$ - factor defined by a shape of a nanoparticle.

The expressions obtained allow us to determine $\sigma_{\mathrm{a}}(\omega, \mathrm{a})$ and $\sigma_{\mathrm{s}}(\omega, \mathrm{a})$ for the next charge carriers transitions: towards quantum size states

$$
\sigma_{a}^{q}(\omega, a)=\frac{8 \pi}{c \hbar} \sum_{i, j} \frac{d_{i j}^{q^{2}} c_{j} \Gamma_{j}}{\left(\left(\omega^{2}-\frac{c_{j}^{2}}{a^{4}}\right)\right)^{2}+\omega^{2} \Gamma_{j}^{2}} \omega^{2}
$$

the cross-sections doesn't exceed $10^{-7}$, so the main factor which defines $\mathrm{T}(\omega, \mathrm{a}, \mathrm{N})$ is a light absorption by nanoparticles.

Fig. 5 shows the light transmission spectrum of $\mathrm{Al}_{2} \mathrm{O}_{3}$ nanoparticles ensemble obtained by using Hitachi U2010 spectrophotometer. Energy gap width of a volume sample of aluminium oxide is within range from $6 \mathrm{eV}$ to $8.8 \mathrm{eV}$, its refraction index is equal to 1.7 approx. Through the use of this sample and a transparent liquid dielectric matrix a heterogenous liquid-phase nanocomposite (HLPN) was prepared. A liquid matrix was based upon the vacuum oil VM-4 (a colorless transparent and viscous liquid with refraction index equal to 1.4 approx.). The vacuum oil has linear optical properties in infrared and visible light. The nanoparticles interacted very weakly, it was possible due to a very low mass concentration of the nanoparticles in the HLPN (0.03\% approx.).

The nanocomposite prepared was placed inside a parallelepiped cuvette made of a $160 \mu \mathrm{m}$ optical glass, cuvette width was $20 \mathrm{~mm}$ along the light propagation direction. The reference sample was made of a similar cuvette with a pure vacuum oil. According to a common procedure we obtained the light transmission spectrum of $\mathrm{Al}_{2} \mathrm{O}_{3}$ nanoparticles ensemble- it was carried out by division of the nanocomposite transmission spectrum by transmission spectrum of the reference sample. Wavelength resolution was $\Delta \lambda=0.5 \mathrm{~nm}$. It follows from the results obtained, that there are two shallow absorption bands within spectral ranges $220-225 \mathrm{~nm}$ and $265-307 \mathrm{~nm}$ as well as relatively deep absorption band within spectral range $308-400 \mathrm{~nm}$. The corresponding minimum values of transmission coefficient $\mathrm{T}(\lambda=237 \mathrm{~nm})=0.425 \quad$ ， $\mathrm{T}(\lambda=287 \mathrm{~nm})=0.445$ and $\mathrm{T}(\lambda=337 \mathrm{~nm})=0.178$. 

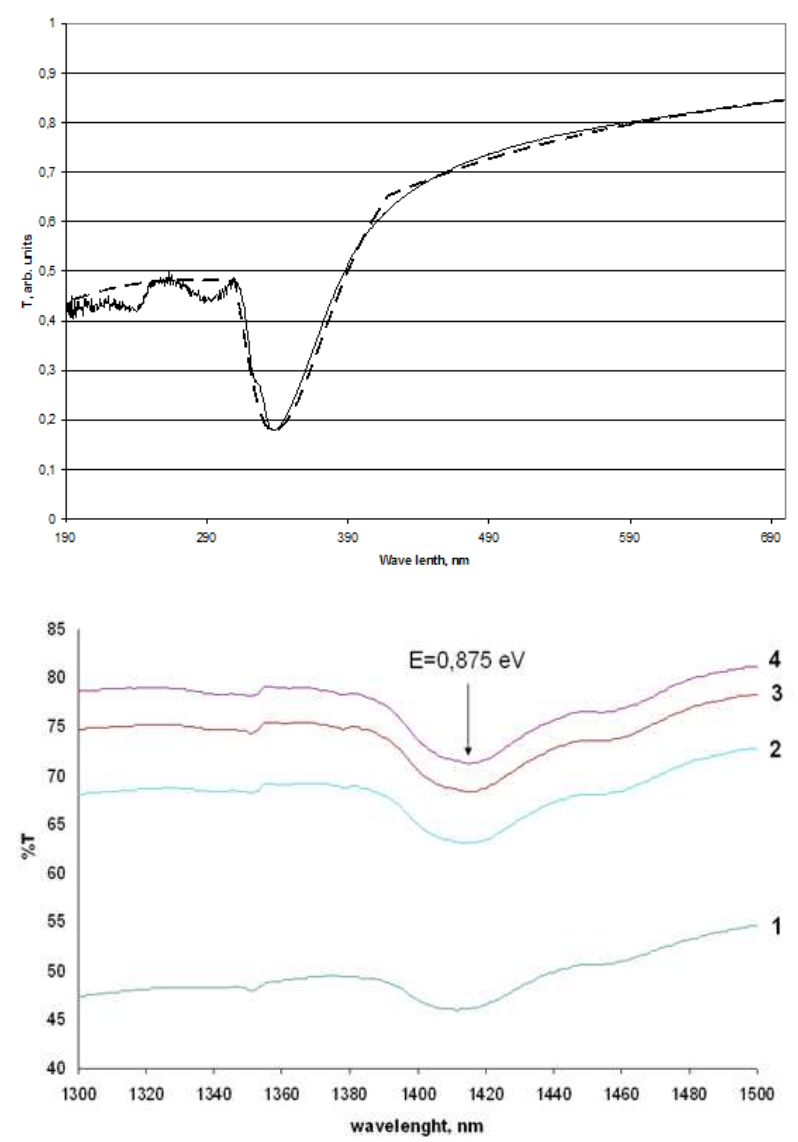

Fig. 5. The light transmission spectrum of $\mathrm{Al}_{2} \mathrm{O}_{3}$ nanoparticles ensemble. Wavelength resolution $\Delta \lambda=0.5 \mathrm{~nm}$. Mass concentration of nanoparticles in the HLPN is $0.03 \%$ approx. a) Infrared light transmission spectrum: experimental (solid line) and theoretical (dashed line) curves. Curves from 1 to 4 correspond to different nanoparticles concentration - from a maximum concentration (curve 1) to a minimum one (curve 2).

The $T(\lambda)$ plot in the vicinity of transmission spectrum minimum (308-400 nm) is of an asymmetrical shape. Long wavelength (in comparison with central wavelength $\lambda=$ $337 \mathrm{~nm}$ ) portion of absorption band curve is more sloping than that of a short wavelength, it counts in favours of exciton states existence in the nanoparticles under study. Photon energy which corresponds to the minimum transmission wavelength $\lambda=337 \mathrm{~nm}$ is equal to $3.7 \mathrm{eV}$. It is considerably less than $E_{g}$ energy gap width of a bulk crystal, but is approximately equal to the value of $\mathrm{E}_{\mathrm{g}}=3.6$ $\mathrm{eV}$ for $\mathrm{Al} 2 \mathrm{O} 3$ nanoparticles stated in the appropriate work [12]. Difference between these values and the values of $E_{g}$ of a bulk crystal are attributable to a considerable change of electronic structure of $\mathrm{Al}_{2} \mathrm{O}_{3}$ crystals caused by small size of nanoparticles and a substantial number of nearsurface defects of nanoparticle's crystal structure due to the complex structure of nanoparticles. In this case the value of $\mathrm{E}_{\mathrm{g}}=3.7 \mathrm{ev}$, and it will correspond with the energy of a transition from defect zone centre to conduction band. Photon energies associated with the other minima equal to $4.1 \mathrm{eV}$ and $5.2 \mathrm{eV}$ correspond to transitions to quantum-size states and energy of $0.875 \mathrm{eV}$ corresponds to a transition between quantum-size states with minimal energy (Fig. 4).

By using the expressions $(8,8 \mathrm{a}-11)$ the model of 5 the $\mathrm{A} 12 \mathrm{O} 3$ nanoparticles ensemble transmission spectrum which relates the transmission spectrum to the light frequency and a size of nanoparticles was developed. Depending upon the light frequency the expression for transmission spectrum has the next form

$$
\begin{aligned}
& \mathrm{T}(\omega, \mathrm{a}) \approx \exp \left[-\mathrm{Nh}\left(\mathrm{A}_{1}^{\mathrm{q}} \omega^{-2}+\mathrm{A}_{1}^{\mathrm{ex}} \mathrm{a}^{2} \omega^{-2}+\mathrm{A}_{1}^{\mathrm{c}} \mathrm{a} \omega\right)\right], \text { when } \omega>\omega_{\mathrm{j}}, \\
& \mathrm{T}(\omega, \mathrm{a}) \approx \exp \left[-\operatorname{Nh}\left(\mathrm{A}_{3}^{\mathrm{q}} \mathrm{a}^{8} \omega^{2}+\mathrm{A}_{3}^{\mathrm{ex}} \mathrm{a}^{2} \omega^{2}+\mathrm{A}_{3}^{\mathrm{c}} \mathrm{a} \omega\right)\right], \text { when } \omega<\omega_{\mathrm{j}} .
\end{aligned}
$$

In the vicinity of resonance frequency $\omega_{\mathrm{j}}(\lambda=337 \mathrm{~nm})$, introducing the quntity $\Delta \omega=\omega-\omega_{\mathrm{j}}$, we obtain

$$
\mathrm{T}(\omega, \mathrm{a}) \approx \exp \left[\left[-\mathrm{Nh} \sum_{\mathrm{i}, \mathrm{j}}\left(\frac{\mathrm{d}_{\mathrm{ij}}^{\mathrm{q} 2} \mathrm{c}_{\mathrm{j}} \Gamma_{\mathrm{j}}}{4 \Delta \omega^{2}+\Gamma_{\mathrm{j}}^{2}}+\frac{\mathrm{d}_{\mathrm{ij}}^{\mathrm{ex} 2} \omega_{\mathrm{j}} \Gamma_{\mathrm{j}} \mathrm{a}^{2}}{4 \Delta \omega^{2}+\Gamma_{\mathrm{j}}^{2}}+\mathrm{A}_{2}^{\mathrm{c}} \mathrm{a} \omega\right)\right)\right]
$$

where summation is carried out over the all state $|i\rangle$ to state $|j\rangle$ transitions.

It was supposed that the main mechanisms responsible for the transmission spectrum witnessed were the charge carriers transitions from defect energy zone, localized within forbidden band, to the exciton states ( $\lambda>337 \mathrm{~nm}$ ), with their subsequent light-induced destruction and the transition to quantum-size states. In case of wavelength $\lambda<337 \mathrm{~nm}$ the main mechanisms are the charge carriers transition from additional allowed energy bands to a conduction band including quantum-size states. The intersections of curves correspond to an experimentally obtained values of light transmission spectrum which were used to derive the set of equations in unknowns $A$ and $\Gamma$.

As it follows from the Fig. 5 there is a good correspondence between experimental curve and the theoretical one which counts in favor of proposed mechanisms of generation of the light transmission spectrum reasoned by the electronic structure of dielectric nanoparticles. It should be noticed that the same transmission spectra can be observed in case of using many other semiconductor and dielectric nanoparticles [12].

\section{Basics of Theory of Optical Nonlinearity of Dielectric Nanocomposites}

Over the last years, experimental studies of nonlinear optical properties of nanocomposites [1-8] with small concentrations of wide band semiconductor and dielectric nanoparticles embedded into dielectric matrix have revealed, that these media, influenced by either of nanosecond pulses and continuous illumination of near infrared and visible light, demonstrate a low threshold (pulse regime $\mathrm{P}_{\mathrm{thr}}<0.5 \mathrm{~nJ} / \mathrm{cm}^{2}$ ) response. This response disappears when the light intensity becomes high, and the dependence of intensity of the output light (passed through the nanocomposite) on the input light (incident upon the 
nanocomposite) intensity $\mathrm{P}_{\text {out }}\left(\mathrm{P}_{\text {in }}\right)$ becomes a linear one. Secondly, despite the broad energy gap of volume samples of nanoparticles, e.g. $\mathrm{Al}_{2} \mathrm{O}_{3}$ has this gap equal to $7.2 \mathrm{eV}$, the nonlinear response can be witnessed in near infrared and visible light ranges. Thirdly, this response exists when the light transmission spectrum of nanoparticles has broad light absorption bands which are absent in volume samples, and there exists such frequency $\omega_{p}$ of the light that is, being different from the central frequency of absorption band, responsible for change of a sign of nonlinear addition to the nanocomposite's refraction index when this frequency is overcome.

The theory of optical nonlinearity of effective refraction index of dielectric nanocomposites in the field of a weak laser radiation is based upon the next assumptions: the main reasons of low threshold nonlinearity of refraction index are: 1) a dipole electrical moment of a nanoparticle, induced by an external optical radiation and caused by the difference of charge carrier states populations which is distinct from the thermal one; 2) the direction of polarization vector of nanoparticles along the polarization vector of the external optical field. The most simple, from the point of view of describing media with nonlinear optical properties, is a «gas» of nanoparticles. Liquid and solid nanocomposites have a more complex structure but they can be prepared easier. Theoretical descriptions of refraction indices of these media have very much in common. Because of this, we consider a nanocomposite which consists of dielectric nanoparticles embedded into transparent liquid matrix made of isotropic dielectric material. The nanocomposite obtained is characterized by linear optical properties and a small coefficient of viscosity of nanoparticle. Let the amount $\mathrm{N}$ of particles per unit volume be small enough to neglect the interaction between nanoparticles. Let $\alpha=\left\{\alpha_{\mathrm{ij}}\right\}$-effective polarization tensor of nanoparticle in such matrix, so the components of electric dipole moment of the nanoparticle, which is induced by a monochromatic and linearly polarized electromagnetic field with frequency $\omega$ and vector of electric field intensity, are defined directly by the external field rather than by the local one. Let introduce two coordinate systems with the common centre. The first one is a coordinate system of principal axes of polarization tensor of an arbitrary nanoparticle $\left\{\alpha_{1}, \alpha_{2}, \alpha_{3}\right\}$ with $\left(\overrightarrow{\mathrm{n}}_{1}, \overrightarrow{\mathrm{n}}_{2,} \overrightarrow{\mathrm{n}}_{3}\right)$ basis vectors, and the second one is a laboratory Cartesian coordinate system with $\left(\overrightarrow{\mathrm{n}}_{x}, \overrightarrow{\mathrm{n}}_{\mathrm{y}}, \overrightarrow{\mathrm{n}}_{\mathrm{z}}\right)$ basis vectors. The $\left\{\alpha_{1}, \alpha_{2}, \alpha_{3}\right\}$ coordinate system defines polarization vector of the nanoparticle as $\overrightarrow{\mathrm{P}}=\sum_{j}^{3} \alpha_{i j}\left(\overrightarrow{\mathrm{n}}_{\mathrm{j}} \overrightarrow{\mathrm{E}}\right) \overrightarrow{\mathrm{n}}_{\mathrm{j}}$. If $\vec{E}$ vector is aligned with $\mathrm{Z}$ axis so the projection of the polarization vector upon this axis is equal to

$$
P_{z}=\sum_{j}^{3} \alpha_{j j} E\left(\vec{n}_{j} \vec{n}_{z}\right)^{2}=\sum_{j}^{3} \alpha_{j j} E \cos ^{2} \theta_{j}
$$

Where $\theta_{1}, \theta_{2}, \theta_{3}$ - angles between vector $\overrightarrow{\mathrm{E}}$ and $\left\{\alpha_{1}, \alpha_{2}, \alpha_{3}\right\}$ axis correspondingly. In the lab coordinate system $\mathrm{P}_{\mathrm{z}}=\alpha_{\mathrm{zz}} \mathrm{E}$. Comparing this expression with the Eq.(16) we obtain $\alpha_{z z}=\left(\alpha_{11} \cos ^{2} \theta_{1}+\alpha_{22} \cos ^{2} \theta_{2}+\alpha_{33} \cos ^{2} \theta_{3}\right)$.

Polarizabilty tensor $\chi=\left\{\chi_{\mathrm{ij}}\right\}$ of such medium to a first approximation can be assumed diagonal and polarization vector of voiume unit is $\overrightarrow{\mathrm{D}}=\chi_{\mathrm{zz}} \overrightarrow{\mathrm{E}}$. In this expression $\chi_{\mathrm{zz}}=\mathrm{N}\left\langle\alpha_{\mathrm{zz}}\right\rangle$ where brackets mean averaging over angles of directions of nanoparticle's polarization vector. Making little manipulations and taking into account the expression $1=\cos ^{2} \theta_{1}+\cos ^{2} \theta_{2}+\cos ^{2} \theta_{3}$ one can obtain the next expression for $-\chi_{z z}=\mathrm{N}\left(\alpha_{0}+\Delta \alpha_{1} \mathrm{Q}_{1}+\Delta \alpha_{2} \mathrm{Q}_{2}\right)$ where $\mathrm{Q}_{1}=\left\langle\operatorname{Cos}^{2} \theta_{1}-\frac{1}{3}>\right.$ and $\mathrm{Q}_{2}=<\operatorname{Cos}^{2} \theta_{2}-\frac{1}{3}>$ actually are parameters of orientation order of nanoparticles in the external field, and $\alpha_{0}=\frac{\alpha_{11}+\alpha_{22}+\alpha_{33}}{3}, \Delta \alpha_{1}=\alpha_{11}-\alpha_{33}$, $\Delta \alpha_{2}=\alpha_{22}-\alpha_{33}$.

Nanoparticle's reorientation in the external field is connected with an alteration of its energy $\mathrm{U}=-\frac{1}{2} \operatorname{Re}\left(\overrightarrow{\mathrm{p}} \cdot \overrightarrow{\mathrm{E}}^{*}\right)$ of interaction with this field. It causes the angle distribution function and hence order parameters $\mathrm{Q}_{1}$ and $\mathrm{Q}_{2}$ to be dependent upon the field intensity, and, through the mediation of $\alpha_{i j}$ component, upon the light frequency. The values of these parameters lie within range from $-1 / 3$ to $2 / 3$ independently of light frequency and its intensity. In case of uniform angular distribution these values are equal to zero. If a one of the $\mathrm{Q}$ parameters is equal to $2 / 3$ it indicates that vector $\vec{p}$ of nanoparticle's dipole moment is directed along the one axis $\left(\mathrm{Q}=\frac{2}{3}\right)$ and is directed perpendicularly the other ones $\left(\mathrm{Q}=-\frac{1}{3}\right)$. In the frequency range where light transmission spectra of nanoparticles have broad absorption bands or sets of relatively narrow bands, the components of tensor $\alpha_{i j}$ should reach maximum values associated with charge carriers dipole state $|\mathrm{n}\rangle$ to state $|\mathrm{g}\rangle$ transitions. It is known that diagonal elements of the $\alpha_{i j}$ tensor within the abovementioned frequency range can be represented as [23].

$$
\alpha_{\mathrm{ii}}(\omega)=\sum_{\mathrm{n}<\mathrm{g}} \sum_{\mathrm{g}} \frac{|\langle\mathrm{n}|\mathrm{er}| \mathrm{g}\rangle|^{2}}{\hbar\left(\omega-\omega_{\mathrm{ng}}+\mathrm{i} \Gamma_{\mathrm{ng}}\right)} \Delta \rho_{\mathrm{ng}},
$$

where summation is carried out over all allowed optical transitions of nanoparticle's charge carriers with transition frequency $\omega_{n g}$, half-width of the transition line $\Gamma_{n g}$ and component of dipole electric moment equal to $\mathrm{p}_{\mathrm{ng}}^{\mathrm{i}}=<\mathrm{n}\left|\mathrm{er}_{\mathrm{i}}\right| \mathrm{g}>$. Population difference $\Delta \rho_{\mathrm{ng}}$ between 
states $|\mathrm{n}\rangle$ and $|\mathrm{g}\rangle$ induced by a light is a function of the incident light intensity . To a double-particles system approximation [21] this function is

$$
\Delta \rho_{\mathrm{ng}}(\mathrm{I})=\left(1-\sum_{\mathrm{n}} \sum_{\mathrm{g}} \frac{\mathrm{I} / \mathrm{I}_{\mathrm{s}}}{\left(\omega-\omega_{\mathrm{ng}}\right)^{2}+\Gamma_{\mathrm{ng}}^{2}\left(1+\mathrm{I} / \mathrm{I}_{\mathrm{s}}\right)} \Gamma_{\mathrm{ng}}^{2}\right) \Delta \rho_{\mathrm{ng}}^{0},
$$

$$
\mathrm{n}(\omega, \mathrm{I}) \approx \mathrm{n}_{0}+\frac{2 \pi \chi_{\mathrm{zz}}(\omega, \mathrm{I})}{\mathrm{n}_{0}}=\mathrm{n}_{0}+\sum_{\mathrm{n}} \sum_{\mathrm{g}} \Delta \rho_{\mathrm{ng}}(\omega, \mathrm{I})\left[\frac{\omega-\omega_{\mathrm{ng}}}{\left(\omega-\omega_{\mathrm{ng}}\right)^{2}+\Gamma_{\mathrm{ng}}^{2}}+\mathrm{i} \frac{\Gamma_{\mathrm{ng}}}{\left(\omega-\omega_{\mathrm{ng}}\right)^{2}+\Gamma_{\mathrm{ng}}^{2}}\right] \mathrm{A}_{\mathrm{ng}}\left(\mathrm{Q}_{1}, \mathrm{Q}_{2}\right),
$$

where

$\mathrm{A}_{\mathrm{ng}}\left(\mathrm{Q}_{1}, \mathrm{Q}_{2}\right)=\frac{2 \pi \mathrm{N}}{\hbar \mathrm{n}_{0}}\left[\frac{1}{3}\left|\overrightarrow{\mathrm{p}}_{\mathrm{ng}}\right|^{2}+\mathrm{Q}_{1}\left(\left|\mathrm{p}_{\mathrm{ng}}\right|^{2}-\left.\left|\mathrm{p}_{\mathrm{ng}}\right|^{3}\right|^{2}\right)+\mathrm{Q}_{2}\left(\left|\mathrm{p}_{\mathrm{ng}}\right|^{2}-\left.\left|\mathrm{p}_{\mathrm{ng}}\right|^{3}\right|^{2}\right)\right]$ is a monotonic rising function of $\mathbf{I}$. Isotropic nanoparticles have a polarization vector always directed along the vector $\overrightarrow{\mathbf{E}}$ and a $\mathrm{A}_{\mathrm{ng}}\left(\mathrm{Q}_{1}, \mathrm{Q}_{2}\right)$ of a maximum value, which is equal where $\Delta \rho_{\mathrm{ng}}^{0}$ - equilibrium thermal population difference, $I_{s}$ - saturation intensity associated with $\frac{\Delta \rho_{0}}{2}$ amount of charge carriers transitioned from state $|\mathrm{n}\rangle$ to state $|\mathrm{g}\rangle$.

Complex nonlinear refraction index $\mathrm{n}(\omega, \mathrm{I})$ of a nanocomposite in the field of linearly polarized light, in view of Eq. (17) and (18), can be expressed as

$$
\mathrm{n}^{\prime}(\omega, \mathrm{I})=\mathrm{n}_{0}^{\prime}+\sum_{\mathrm{n}} \sum_{\mathrm{g}}\left\{\left[1-\left(\frac{\left(\mathrm{I} / \mathrm{I}_{\mathrm{s}}\right) \Gamma_{\mathrm{ng}}^{2}}{\left(\omega-\omega_{\mathrm{ng}}\right)^{2}+\Gamma_{\mathrm{ng}}^{2}\left(1+\mathrm{I} / \mathrm{I}_{\mathrm{s}}\right)}\right)\right] \frac{\mathrm{A}_{\mathrm{ng}}\left(\mathrm{Q}_{1}, \mathrm{Q}_{2}\right)\left(\omega-\omega_{\mathrm{ng}}\right)}{\left(\omega-\omega_{\mathrm{ng}}\right)^{2}+\Gamma_{\mathrm{ng}}^{2}} \Delta \rho_{\mathrm{ng}}^{0}\right\} .
$$

Electronic structure of dielectric nanoparticles is characterized by broad light absorption bands, absent within volume samples, a wide energy gap, a subzone of allowed energy (exciton, doped, etc.) of electrons within forbidden band and adjacent to the bottom of conduction band and broadened quantum-size levels (minizones). Allowance of the electronic structure of nanoparticles can be made by substituting in the Eq. (5) the integration within limits from $\left(\omega-\Delta \omega_{1}\right)$ to $\left(\omega+\Delta \omega_{2}\right)$ with state densities $g_{1}$ and $g_{2}$ correspondingly for the summation over $|g\rangle$ states. Here $\omega_{n}$ is the frequency of interzone

$$
\mathrm{n}^{\prime}(\omega, \mathrm{I})=\mathrm{n}_{0}^{\prime}+\frac{\hbar}{2} \sum_{\mathrm{n}} \mathrm{A}_{\mathrm{n}}\left(\mathrm{Q}_{1}, \mathrm{Q}_{2}\right) \Delta \rho^{0}\left[\mathrm{~g}_{1} \ln \frac{\left(\omega-\left(\omega_{\mathrm{n}}-\Delta \omega_{1}\right)\right)^{2}+\Gamma_{\mathrm{n}}^{2}\left(1+\frac{\mathrm{I}}{\mathrm{I}_{\mathrm{s}}}\right)}{\left(\omega-\omega_{\mathrm{n}}\right)^{2}+\Gamma_{\mathrm{n}}^{2}\left(1+\frac{\mathrm{I}}{\mathrm{I}_{\mathrm{s}}}\right)}+\mathrm{g}_{2} \ln \frac{\left(\omega-\omega_{\mathrm{n}}\right)^{2}+\Gamma_{\mathrm{n}}^{2}\left(1+\frac{\mathrm{I}}{\mathrm{I}_{\mathrm{s}}}\right)}{\left(\omega-\left(\omega_{\mathrm{n}}+\Delta \omega_{2}\right)\right)^{2}+\Gamma_{\mathrm{n}}^{2}\left(1+\frac{\mathrm{I}}{\mathrm{I}_{\mathrm{s}}}\right)}\right] \text {. }
$$

In case of nanoparticles a value of $\Gamma_{n}$ can be much higher than $\Gamma_{n g}$ as in case of molecules. This is supported by the fact of considerable broadening of absorption bands in nanoparticles in comparison with that of volume samples. Eq. (21) shows that nonlinear part of refraction index $\mathrm{n}_{\mathrm{n}}(\mathrm{I})$, being the function of external illumination intensity, is described by product of $A_{n}\left(Q_{1}, Q_{2}\right)$ and logarithms within square brackets. The behavior of these multipliers with increase of light intensity is the opposite one as it shown in Fig. 6. transition from state $|n\rangle$ to a quantum-size zone, $\Delta \omega_{1}$ the width of allowed energies subzone within forbidden band, $\Delta \omega_{2}$ - the width of subzone of quantum size levels which corresponds to $|\mathrm{g}\rangle$ states. For the sake of simplicity of the expressions to obtain, let us assume the state densities and to be independent of frequency and $\Gamma_{n g}=\Gamma_{n}$. Going from summation to integration over frequencies in Eq. (20) we obtain 
have $A_{n}\left(Q_{1}, Q_{2}\right)$ of a maximum value. When the light intensity increases, the value of $A_{n}\left(Q_{1}, Q_{2}\right)$ rises up rapidly (Fig. 1a) reaching its maximum (corresponding with a full orientation of nanoparticles along the field) and the values of logarithms in the nonlinear part of the refraction index $n^{\prime}(\omega, I)$ go down to zero (Fig. 6a). From the physical point of view it corresponds with population differenc $\Delta \rho_{\text {ng }}$ e equal to zero.

The competition between these two opposedly directed processes leads to a rather rapid increase of nonlinear part of refraction index of a nanocomposite, when the external illumination intensity grows up, reaching up its maximum with a subsequent fall down to zero (Fig. 6b). During experiments it was discovered that a similar dependence of refraction index emerged as a selfrefraction of the laser beam, and a nonlinearity of dependence of the output light intensity on the input light intensity $\mathrm{P}_{\text {out }}\left(\mathrm{P}_{\text {in }}\right)$. The next important result follows from the Eq. (21). If $\left(\omega-\omega_{1}\right)>0$ than the nonlinear part of refraction index is positive, if $\left(\omega-\omega_{1}\right)<0$ so such light frequency $\omega_{p}$ will exist, that will be responsible for the change of sign of nonlinear part when this frequency is exceeded. In particular, if $g_{1}=g_{2}$, so $\omega_{\mathrm{p}}=\omega_{\mathrm{n}}-\frac{\Delta \omega_{1}-\Delta \omega_{2}}{2}$ and if $\omega>\omega_{p}$ it has a negative value. This effect of a sign changing was observed during experiments described in works $[3,4]$ when the frequencies corresponded to (2-2.3) $\mathrm{eV}$ were used. According to Eq. (21), the change of sign must occur when the difference $\left(\Delta \omega_{1}-\Delta \omega_{2}\right)$ corresponds to 3-3.4 eV. According to works [7-10,12], in this case $\omega_{\mathrm{n}}$ is equal to $3.7 \mathrm{eV}$, and absorption band has a very smear longwavelength edge.

The other experimentally discovered fact follows from the Eq.(21). When $\left(\omega-\omega_{\mathrm{n}}\right)>\left(\Delta \omega_{1}, \Delta \omega_{2}\right)$ the refraction index of the nanocomposite is linear. It means that low threshold nonlinear optical response is observed within the frequency range inside the absorption band. We can refer to the only one work [24] which contains indirect estimation of nonlinear addition to the refraction index $\mathrm{n}_{\mathrm{n}}^{\mathrm{ex}}$ based upon experimental results. Thus, for the purpose of comparison of theoretical values of $n_{n}$, we use the experimental results described in works [7,24] which allow us to make an estimation of $\mathrm{n}_{\mathrm{n}}^{\mathrm{ex}}$. The abovementioned experiments shown a photoinduced nonthermal selfrefraction of the laser beam passing through a cuvette with a liquid nanocomposite as well as formation of interference rings of number $M$ which is sufficient for the estimation of a nonlinear addition to a refraction index. The experimental results of the work [7] demonstrate from 2 to 4 bright rings in dependence on the $633 \mathrm{~nm}$ light intensity. If the nanocomposite layer thickness $\ell$ is equal to $20 \mathrm{~mm}$ so, by using the $-\mathrm{M}=\frac{\left|\mathrm{n}_{\mathrm{n}}^{\mathrm{ex}}\right| \ell}{\lambda} \quad$ it is easy to determine that $\mathrm{n}_{\mathrm{n}}^{\mathrm{ex}} \approx 0.13 \cdot 10^{-3}$ for $\mathrm{M}=4$ and $\mathrm{n}_{\mathrm{n}}^{\mathrm{ex}} \approx 0.65 \cdot 10^{-4}$ for $\mathrm{M}=2$. The nanoparticles concentration is $\mathrm{N} \approx 10^{13} \mathrm{~cm}^{-3}$ and their size varies from 40 to $50 \mathrm{~nm}$. The matrix refraction index $\mathrm{n}_{0} \approx 1.4$, the refraction index of $\mathrm{Al}_{2} \mathrm{O}_{3}$ nanoparticles is 1.7 approx. Photon energy corresponding to the maximum light absorption by nanoparticles is $3.7 \mathrm{eV}$ (Fig. 5), and the energy quantum of laser radiation is equal to $1.96 \mathrm{eV}$. In this case a dipole transition of electron to an exciton state is most probable due to the quantum energy which is less than energy gap of the nanoparticle. Due to its small volume $\left(10^{-17} \mathrm{~cm}^{-3}\right)$, the nanoparticle will host only a small quantity of such transitions. Thus, we use the Eq.(20) to estimate the value of $\mathrm{n}_{\mathrm{n}}$.

For small intensities of laser radiation and a small amount of possible transitions we have $\mathrm{n}_{\mathrm{n}}(\mathrm{I}) \approx \frac{\mathrm{A}_{\mathrm{ng}}\left(\mathrm{Q}_{1}, \mathrm{Q}_{2}\right)\left(\omega-\omega_{\mathrm{ng}}\right)}{\left(\omega-\omega_{\mathrm{ng}}\right)^{2}+\Gamma_{\mathrm{ng}}^{2}} \Delta \rho_{\mathrm{ng}}^{0}$, where $\Delta \rho_{\mathrm{ng}}^{0} \approx 1$. The dipole electric moment, induced by transition, for such nanoparticles is proportional to their size $\mathrm{p}_{\mathrm{ng}} \approx \Lambda$ ea where form-factor $\Lambda \approx 1$. The nanoparticles used in the experimental studies [3] were of wide absorption band; wavelength $\lambda=633 \mathrm{~nm}$ lies within absorption band, so the $\omega_{\mathrm{ng}}$ is equal to central frequency corresponding to $\lambda=335 \mathrm{~nm}$ wavelength. And $\left(\omega-\omega_{\mathrm{ng}}\right)^{2} \approx \Gamma_{\mathrm{ng}}^{2}$. Using the expression for $\mathrm{A}_{\mathrm{ng}}\left(\mathrm{Q}_{1}, \mathrm{Q}_{2}\right)$ we obtain $\mathrm{n}_{\mathrm{n}} \approx 0.32 \cdot 10^{-4}$ for small light intensities $\left(\mathrm{Q}_{1}\right.$ and $\mathrm{Q}_{2}$ are close to zero) and $\mathrm{n}_{\mathrm{n}} \approx 0.96 \cdot 10^{-4}$ with a full orientation of nanoparticles along the field. The nanocomposite described in work [24] was based upon the immersion of quantum dotes $\mathrm{CdSe} / \mathrm{ZnS}$ in toluene with sizes of $1.9 \mathrm{~nm}$ and 2.6 $\mathrm{nm}$ with a concentration $1014 \mathrm{~cm}-3$ approx. Similar to the previous case, laser radiation frequencies were inside the limits of the absorption bands and $\left(\omega-\omega_{\mathrm{ng}}\right) \approx\left(10^{12} \div 10^{13}\right) \mathrm{Hz}$. Theoretically estimated values of nonlinear addition to the refraction index were $\mathrm{n}_{\mathrm{n}} \in\left[8 \cdot 10^{-5} \div 1.3 \cdot 10^{-3}\right]$, the values defined experimentally, were described in the work and were $\mathrm{n}_{\mathrm{n}}^{\mathrm{ex}} \in\left[8.75 \cdot 10^{-5} \div 3.5 \cdot 10^{-3}\right]$. Due to limitation of the paper we have closely considered only nonlinear refraction with no regard to light absorption and scattering, which can be investigated by using the methods discussed above.

A good qualitative and quantitative correspondence of theoretical model proposed with experimental data makes it possible to say that low threshold nonlinear optical response of dielectric nanostructures and its behavior ,with the growth of the external illumination intensity, are caused, mainly, by photoinduced dipole electric moment of nanoparticles and by dependence charge carriers population difference on light intensity as well as their 
orientation along the polarization vector of the external optical field.

We have to notice that we didn't take into account a mechanisms, additional to regarded ones, which consists of spatial redistribution of nanoparticles in the field of a laser beam. This mechanisms can be effective in the constant laser fields with their high irregularity along the beam profile and a high mobility of nanoparticles inside the matrix.

\section{Nonlinear Optical Effects in the Laser Field: Experiment}

\subsection{Experimental Setup}

For investigation of the influence of optical properties of the liquid phase matrix upon nonlinear optical properties of heterogeneous liquid-phase nanocomposites (HLPN) the experimental setup shown in Fig. 7 was used [7]. As a source of continuous illumination we used a $50 \mathrm{~mW} \mathrm{He}-\mathrm{Ne}$ laser (1) at the wavelength of $632 \mathrm{~nm}$ pumped by array of $40 \mathrm{~mW}$ continuous semiconductor YAG:Nd lasers at the wavelength of a second harmonic $\lambda=532 \mathrm{~nm}$. The laser power was controlled by a mounted set of neutral light filters (2). In order to control the radiation power level a part of the laser beam was separated by a beam splitter (3) and was registered by a photodetector (9); a signal of the photodetector after passing through an ADC (3) was received by a PC (11). A $30 \mathrm{~cm}$ focal length lens (4) was used for focusing the laser beam on the plane-parallel cuvette (5) with the HLPN under study. The cuvette used was made of $160 \mu \mathrm{m}$ optical glass and was of a length of $20 \mathrm{~mm}$ along the light propagation direction. An axially mounted diaphragm with the aperture of $1 \mathrm{~mm}$ diameter separated a central area of the laser beam to be registered by the photodetector (7). The registered signal was digitalized by ADC (7) and processed by the PC (11).

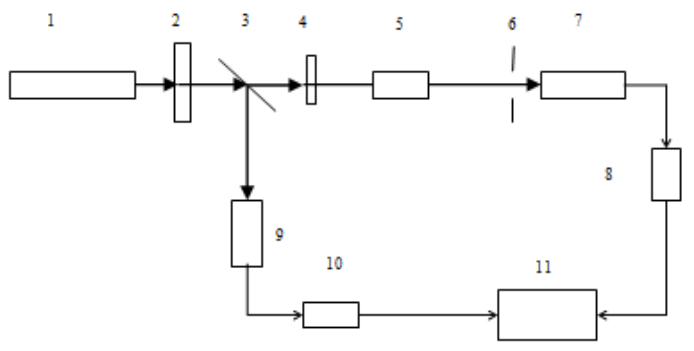

Fig. 7. The scheme of experimental setup

As liquid phase matrices of HLPN the next transparent media were used: vacuum oil VM-4 (refraction index $\mathrm{n} \sim 1,4)$ and immersion oil $(\mathrm{n} \sim 1,5)$ with linear and nonlinear optical properties in visible and near infrared ranges of light correspondingly. Nanoparticles in the HLPN were of wide band dielectric $\mathrm{Al}_{2} \mathrm{O}_{3}$ (corundum) with $\mathrm{n} \sim 1.7$. A corundum crystal has a hexagonal symmetry and its energy gap width, according to different sources, varies from 6.26 to $8.8 \mathrm{eV}[9,10]$. The nanoparticles of a current shape were extracted from industrially prepared by the explosion method nanopowders by levigating in the acetone. Volume concentration of nanoparticles in HLPN was varied from $0.03 \%$ to $0.3 \%$. Maximal average lateral dimension of nanoparticles was within range from 40 to 50 $\mathrm{nm}$ and the surface of $\mathrm{Al}_{2} \mathrm{O}_{3}$ nanoparticles isn't flat but of a complex multipeak pyramidal form (Fig. 3).

\subsection{Single-Frequency Light-HLPN Interaction}

To estimate the influence of nanoparticles upon the refraction index of HLPN experimentally, a liquid-phase matrix based upon the vacuum oil VM-4 was used. The oil, according to technical conditions, was of $\mathrm{dn} / \mathrm{dT} \mid \approx 0$ degree $^{-1}$. Fig.8 shows normalized dependencies of paraxial part of light radiation passed through HLPN $\left(\mathrm{I}_{\text {out }} / \mathrm{I}_{0}\right)$ - upon input light intensity $\mathrm{I}_{\text {in }}$ obtained experimentally for wavelengths

$\lambda=633 \mathrm{~nm}$ (curve 1) and $\lambda=532 \mathrm{~nm}$ (curve 2). Volume concentration of $\mathrm{Al}_{2} \mathrm{O}_{3}$ nanoparticles in HLPN was equal to $0.3 \%$.

As it follows from the results obtained, the curves shown if Fig.8, starting from intensity values of $160 \mathrm{~mW} / \mathrm{mm}^{2}$ approx., display nonlinear portions. In case of the light with $\lambda=532 \mathrm{~nm}$ one can observe a limitation of the light passed through HLPN, for the light with $\lambda=633 \mathrm{~nm}$ we see a «bleaching» of the light transmission. When a liquidphase matrix based upon immersion oil with $\mathrm{d} / \mathrm{dT}=-3 \cdot 10^{-3}$ degree $^{-1}$ was used, a thermal mechanism of change in the refraction index was triggered. Fig. 9 displays normalized dependencies of output light intensities $\left(I_{\text {out }} / I_{0}\right)$ on the input light intensity obtained experimentally for both pure immersion oil (wavelength $\lambda=633 \mathrm{~nm}$, curve 1$)$ and HLPN ( $\lambda=633 \mathrm{~nm}$ and $\lambda=$ $532 \mathrm{~nm}$ ) with volume concentration of nanoparticles equal to $0.3 \%$.

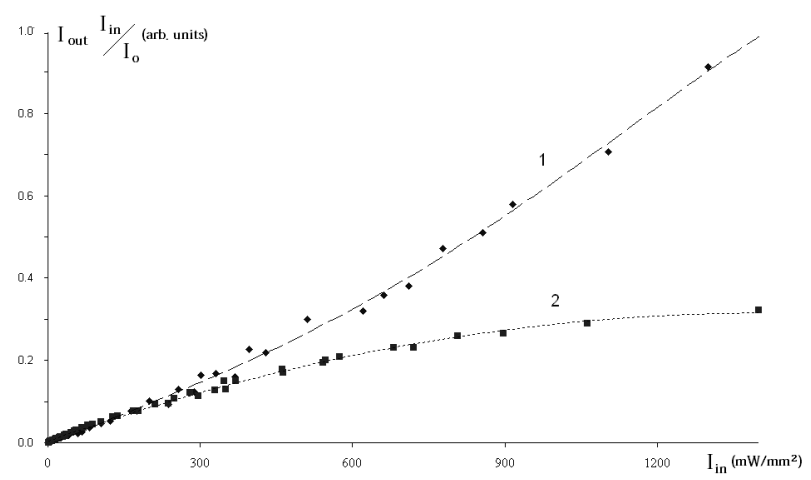

Fig. 8. Dependencies of intensity of the light passed through the VM-4 oil based HLPN on input light intensity (curve $1-\lambda=633 \mathrm{~nm}$, curve 2 $\lambda=532 \mathrm{~nm}, I_{0}=1400 \mathrm{~mW} / \mathrm{mm}^{2}$ ).

As it follows from the Figure, in contrast to the previous case (Fig. 8), this nanocomposite demonstrates the light transmission nonlinearity when light intensities are of 40 $\mathrm{mW} / \mathrm{mm}^{2}$ approx. The other distinguishing feature of this 
nanocomposite is the behavior of curves 1-3 in Fig.9 similar to that of curves 1-3 in Fig.8 for the both wavelengths at the first phase of $\mathrm{I}_{\text {in }}$ growth (up to $300-$ $350 \mathrm{~mW} / \mathrm{mm}^{2}$ ). However, as the input light intensity $I_{\text {in }}$ ( $\lambda=633 \mathrm{~nm}$ ) grows up, further portion of HLPN light transmission curve oscillates, and for the light of $532 \mathrm{~nm}$, after reaching a limitation level, one can observe a considerable decrease in HLPN light transmission. Thus, even the small addition ( $0.3 \%$ approx.) of the nanoparticles to a matrix can cause substantial alterations of optical properties of the medium. In particular, limitation level of light intensity for HLPN is proved to be 6 times smaller.

Oscillations of HLPN light transmission are caused by self-refraction and diffraction smearing of the light with $\lambda=633 \mathrm{~nm}$ along the beam cross-section after the light passing through the HLPN (Fig. 10).

As it follows from the experimental data, matrix material affects considerably upon optical properties of HLPN. Dependently on the wavelength, light transmission nonlinearity can be expressed as a light radiation limitation or as HLPN «bleaching».

The basics of the theory of nonlinear optical properties of dielectric nanocomposite are described in chapter 2. In case of immersion oil matrix one has to take into account the mechanism of formation of a thermal negative cylindrical lens.

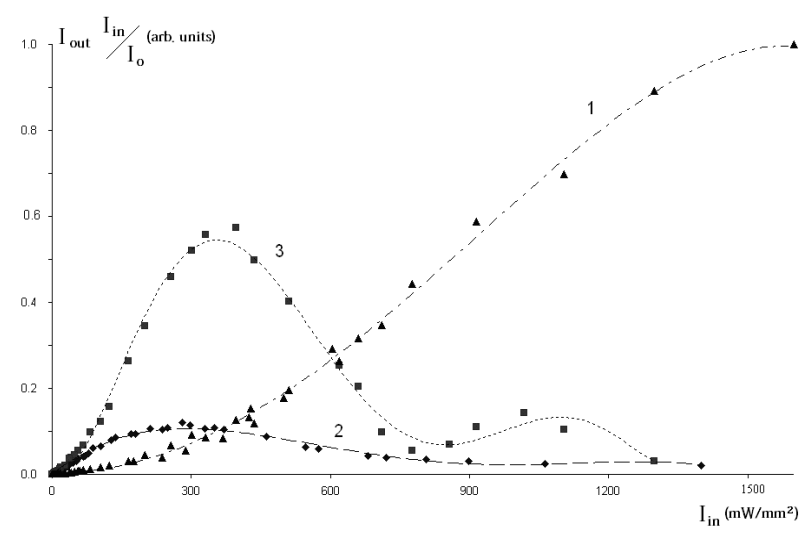

Fig. 9. Dependencies of intensities of light $(\lambda=633 \mathrm{~nm})$ passed through a pure immersion oil (curve 1) and through the HLPN based upon the immersion oil (curve $2-\lambda=532 \mathrm{~nm}$, curve $3-\lambda=633 \mathrm{~nm}$ ) upon the input light intensity, $I_{0}=1600 \mathrm{~mW} / \mathrm{mm}^{2}$.

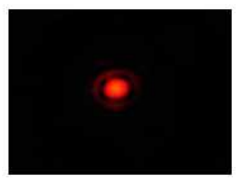

$I=0.1 \mathrm{Wmm}^{2}$

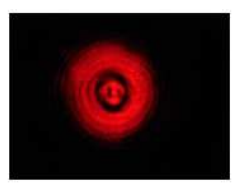

$I=0.8 \mathrm{Wmm}$

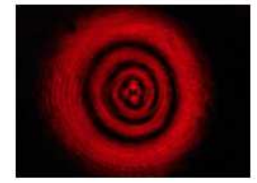

$I=3.12 \mathrm{Wmm}^{2}$
Fig. 10. Light intensity distributions in the far field along the beam crosssection after the light passing through the HLPN based upon immersion oil at the different light intensities $\mathrm{I}_{\mathrm{in}}$ with nanoparticles concentration equal to $0.03 \%$.

For an accurate description of mechanisms of formation of nonlinear optical properties observed, the knowledge of electronic structure of nanoparticles embedded into matrix is required. It is a complex issue with a solution rarely possible to obtain.

However, it follows from the common considerations that a complex shape of a nanoparticle and a higher dielectric permittivity of the nanoparticle's material, in comparison with that of a matrix material, should cause a broadening of exciton band of charge carriers energy levels. When the nanoparticles are illuminated by a light with the wavelengths of $532 \mathrm{~nm}$ and $633 \mathrm{~nm}$, light quanta energy isn't high enough to transmit electrons from the valence band to the conduction band $\left(\mathrm{E}_{\lambda=532}=2,34 \mathrm{eV} ; \mathrm{E}_{\lambda=633}=\right.$ $1,97 \mathrm{eV})$. Therefore light quanta interact with electrons inside the impurity band. Quantum energy of a light with $\lambda=532 \mathrm{~nm}$ is higher than that of a photon with frequency of $\omega_{p}$, which is responsible for change of sign of nonlinear addition to a negative one when being exceeded.

As a result of this processes, a nonlinear negative addition to the refraction index appears. It leads to the formation of a negative gradient lens within the HLPN. It explains the form of the curves 2 in Fig. 8 and 9, and in doing so limitation saturation, proceeding along with the growth of light intensity, is connected with a finite amount of electrons in impurity band as well as approach of photoinduced state population difference $\Delta \rho_{n g}$ of charge carriers to zero.

A frequency of the $633 \mathrm{~nm}$ light is lesser than $\omega_{\mathrm{p}}$ and therefore nonlinear addition to refraction index has a positive sign. When the length of interaction between the light wave and the HLPN is high enough the increase in refraction index can cause a formation of a waveguide channel and additional concentration of laser radiation in the paraxial area. As a result, with the increase of light intensity one can see a «bleaching» of HLPN (curve 1 in Fig.8). Limitation of the «bleaching» for the light of 633 $\mathrm{nm}$ has the same reason as in case of the $532 \mathrm{~nm}$ light.

Utilizing a HLPN based upon immersion oil with $\partial n / \partial T<0$, we observe an additional mechanism of formation of thermal negative cylindrical lens. It causes a faster saturation of the light limitation dependence and its consequent rapid decay with increase of input light intensity (curve 2 in Fig.9). When the light of $633 \mathrm{~nm}$ is passed through HLPN, the increase of refraction index at the initial phase of growth of $I_{\text {in }}$ dominates over its thermal decay, and it leads to a medium «bleaching». Then, owing to a saturation, a process of antiwaveguide formation will be dominating, and it will cause less light transmission of a medium. The saturation process, being an obstacle for light selfrefraction in the medium, as well as subsequent increase in input light intensity, contributes largely to a formation of multilayer waveguides in the composite which it its turn causes a light intensity changes in the paraxial area. This effect allows us to explain the form of the curve 3 shown in Fig. 9. 


\subsection{Double-Frequency Interaction of Collinear Light Laser Beams in HLPN.}

In order to investigate the process of collinear interaction between the laser beams of different wavelengths in heterogeneous liquid-phase nanocomposite the experimental setup shown in Fig. 11 was used [7]. We chose a continuous illumination source based on $50 \mathrm{~mW}$ He-Ne laser (1) at the wavelength $\lambda=632 \mathrm{~nm}$ pumped by array of continuous semiconductor $40 \mathrm{~mW}$ YAG:Nd lasers at the wavelength of a second harmonic $\lambda=532 \mathrm{~nm}$. The laser power was controlled by a mounted set of neutral light filters (3) and (6). In order to control a radiation power level a part of the laser beam was separated by beam splitters (4) and (7) and was registered by photodetectors (5) and (8); signals from the photodetectors after digitalizing with ADC (16) and (17) were processed by the PC (18). Laser beams (1) and (2) were combined collinearly by using a beam splitter (9). A lens (10) with a high focal length $(\mathrm{F}=30 \mathrm{~cm})$ was used for focusing the laser beam upon the cuvette (11) with HLPN.

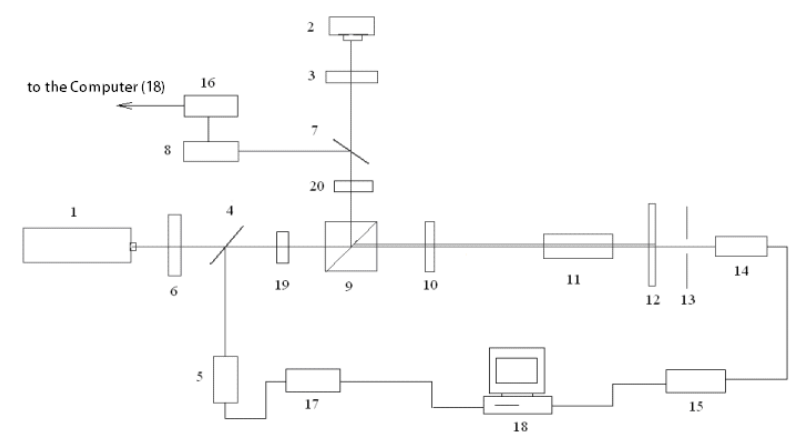

Fig. 11. The scheme of experimental setup

Laser beam diameter in the waist area was of $0.6 \mathrm{~mm}$ approx. The plate-parallel cuvette used in work was made of optical glass and was of a length of $20 \mathrm{~mm}$ along the light propagation direction. Narrow-band interferometric filter (12) was used to separate an illumination of a current wavelength. Point diaphragm (13) with a $1 \mathrm{~mm}$ diameter aperture filtered paraxial area of a laser beam. The passed light was registered by CCD matrix (14) and the light signal after ADC (15) was processed by a PC (18). Mechanical shutters (19) and (20) were used to control a light input into a sample cuvette. During experimental studies liquid phase matrices of HLPN based upon the immersion oil were used. The nanoparticles in HLPN were of wide band dielectric $\mathrm{Al}_{2} \mathrm{O}_{3}$ with an average size of 40 $50 \mathrm{~nm}$ approx. Volume concentration of nanoparticles in HLPN was equal to $0.3 \%$. The experiment was carried out to investigate a dependence of HLPN output light intensity in the paraxial area $\mathrm{I}_{+}\left(\mathrm{I}_{-}\right)$on the intensity of input light with wavelengths of $532 \mathrm{~nm}$ and $633 \mathrm{~nm}$ in case of their collinear co-propagation.

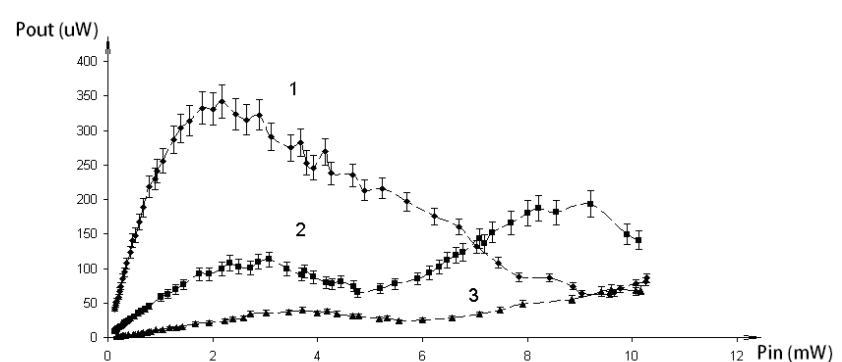

Fig. 12. The dependence of laser output power $(\lambda=633 \mathrm{~nm})$ in the paraxial field on the input power at the different values of the power: curve $1-P_{\text {green }}=2 \mathrm{~mW}$, curve $2-P_{\text {green }}=5 \mathrm{~mW}$, curve $3-P_{\text {green }}=10 \mathrm{~mW}$.

The measurements of $\mathrm{I}_{+}\left(\mathrm{I}_{-}\right)$were carried out after the extinction of transient processes and a stationary regime establishment. The period of transients occur during activation of the second wavelength $(\lambda=532 \mathrm{~nm}$ or 633 $\mathrm{nm}$ ) light was up to $2-2.5 \mathrm{sec}$. Fig. 12 shows experimental dependencies of light transmission $(\lambda=633 \mathrm{~nm})$ at the collinear (control) laser beam $(\lambda=532 \mathrm{~nm})$ power equal to: $2 \mathrm{~mW}$ - curve $1,5 \mathrm{~mW}$ - curve $2,10 \mathrm{~mW}$ - curve 3 . Fig. 13 shows experimental dependencies of light $(\lambda=$ $532 \mathrm{~nm}$ ) transmission at the power of collinear (control) laser beam $(\lambda=633 \mathrm{~nm})$ equal to: $8 \mathrm{~mW}$ - curve $1,4 \mathrm{~mW}$ - curve $2,1 \mathrm{~mW}$ - curve 3 .

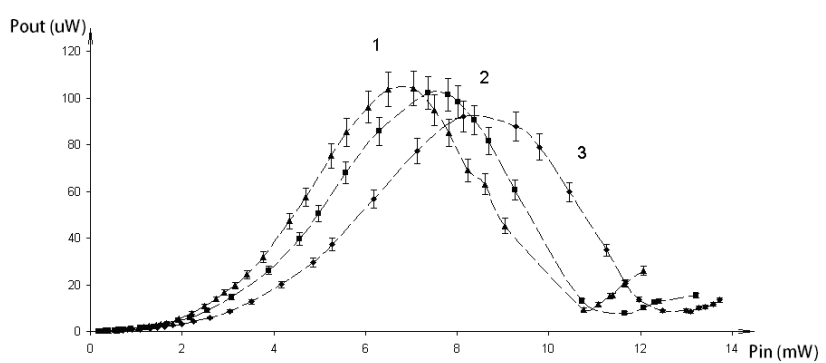

Fig. 13. The dependence of output light $(\lambda=532 \mathrm{~nm})$ intensity at paraxial area of the beam on the input light intensity $(\lambda=532 \mathrm{~nm})$ at the different values of the power: curve $1-P_{\text {red }}=8 \mathrm{~mW}$, curve $2-P_{\text {red }}=4$ $m W$, curve $3-P_{\text {red }}=1 \mathrm{~mW}$.

It follows from the measurement results (Fig.12), that during double-frequency collinear propagation of the light in the course of input light $(\lambda=632 \mathrm{~nm})$ increase the medium light transmission represents itself amplitudedecaying oscillations. In this case maximum values of light transmissions and intensities, associated with these maxima, decrease when the light $(\lambda=532 \mathrm{~nm})$ intensity grows up.

The dependencies of light $(\lambda=532 \mathrm{~nm})$ transmission during a co- propagation of the light $(\lambda=633 \mathrm{~nm})$, obtained at the power range less than $6 \mathrm{~mW}$, don't display such oscillations as it shown in Fig. 13. In both cases one can observe deviation from the linear dependence $\mathrm{I}_{+}\left(\mathrm{I}_{-}\right)$ witnessed at small power of input light $(\lambda=633 \mathrm{~nm})$ equal to $2 \mathrm{~mW}$ (Fig. 12). For the $532 \mathrm{~nm}$ light these values depend upon control light intensity and lie within range from $0.2 \mathrm{~mW}$ to $2 \mathrm{~mW}$ (Fig. 13). 
In case of interaction between collinear laser beams with different wavelengths one can witness interference between induced by these beams nonlinear mechanisms of modulation of optical properties of HLPN. In particular, for the $532 \mathrm{~nm}$ laser beam which interacts with the $633 \mathrm{~nm}$ laser beam in the HLPN on the conditions of frequency dispersion a nonlinear addition $n_{1}$ for the green light can be less than that for the red light; that, being combined with a thermal heating of the medium, cause less power of the green light passed through HLPN at smaller values of input light power. Curves 2-3 shown in Fig. 13 demonstrate this effect.

For the light with $\lambda=633 \mathrm{~nm}$ collinear interaction with the laser beam of $532 \mathrm{~nm}$ wavelength enhances the competition between the two processes: negative and positive lens formations. Due to these processes the maximum transmission of $633 \mathrm{~nm}$ light passing through HLPN is decreased as the $532 \mathrm{~nm}$ light intensity grows up. It is proved with curves 2 and 3 shown in Fig. 12.

As it follows from the results obtained (Fig. 12 and 13), when the light with wavelength of $532 \mathrm{~nm}$ is controlled by the light with wavelength of $633 \mathrm{~nm}$, the size of modulation for collinear beams - the modulated beam and the control one, of current intensities can reach $10 \mathrm{~dB}$. When the $633 \mathrm{~nm}$ light is controlled by the $532 \mathrm{~nm}$ light, the attenuation value can reach $5 \mathrm{~dB}$ approx.

\section{Conclusions}

The results of latest studies of nonlinear optical effects in dielectric nano-phase composites based upon dielectric nanoparticles show that, as a result of interaction between laser radiation and a nanoparticle-matrix complex, one can observe a low-threshold optical nonlinearity which depends upon a liquid-phase matrix material and a concentration of nanoparticles in HLPN.

The observed nonlinearity of the optical properties is caused by a great amount of additional energy levels and allowed energy levels within nanoparticle's forbidden band which are connected with a complex geometrical shape of nanoparticles and with their small sizes.

Being dependent of wavelength of a laser radiation, the light transmission nonlinearity can cause

Depending upon the wavelength of laser radiation, the light transmission nonlinearity can be evident as a light limitation as well as «bleaching» within a limited range of small light intensities, due to a self-refraction or a nonlinear absorption of the light.

The main reasons of this low-threshold optical nonlinearity are: a photo-induced modulation of a medium refraction index during a generation of nonequilibrium charge carriers and a medium polarization caused by laser excitation of charge carriers states, and the effect of light defocusing which, being weakly dependent of the laser radiation wavelength, depends upon liquid-phase matrix material and is reasoned by a dependence of the heterogeneous medium refraction index on the temperature.
The properties of low-threshold nonlinear optical response as well as its behavior with an increase of the external illumination are reasoned, mostly, by value of the photo-induced dipole electric moments of nanoparticles, dependence of population difference of charge carriers states on the light intensity, and the nanoparticles orientations along the external light field polarization vector. The same mechanisms are responsible for effective nonlinear interaction between collinear laser beams of different wavelengths within the HLPN.

The optical nonlinearity, observed in dielectric nanocomposites, allows one to control a nanocomposite light transmission and, correspondingly, to modulate parameters of the light passing through. As a result, one has the way to creation of optical radiation control devices based upon the self-refraction processes, light limitation or bleaching, as well as the collinear nonlinear optical interaction between the laser beams of different wavelengths within the HLPN.

The experiments conducted feature an ability to optimize the light control process by a proper selection of energetic and spectral properties of the controlling laser beam. It opens the way to development of both optically controlled filters for lightwave systems and modulators for input devices of optical processors.

\section{References}

[1] V.P. Dzyuba, A.E. Krasnok, Yu.N. Kulchin. (2010). Nonlinear refractive index of the dielectric nanocomposites in weak optical fields. Technical Physics Letters (Rus), Vol.36, No 21, pp. 1-9, ISSN: 0320-0116

[2] V.P. Dzyuba, Yu.N. Kulchin. (2010). The Variation of the Nanopaticles' Shape and Quantum -Dimensional States of the Quantum Dots or Nanoparticles. Pacific Science Review. V.12, No 1, pp. 102-104, ISSN: 1229-5450

[3] V.P. Dzyuba, A.E. Krasnok, Yu.N. Kulchin, I.V. Dzyuba. (2010). Nonlinear Transmission of Light by Dielectric Nanocomposites. Pacific Science Review. V.12, No 1, pp. 106-108, ISSN: 1229-5450

[4] A.V. Federov. (2005). Optics of nanostructure, Nedra, ISBN: 5-94089-059-8, Saint Petersburg

[5] Litty Irimpan, Bindu Krishnan, V.P.N. Nampoori, P. Radhakrishnan. (2008) Nonlinear optical characteristics of nanocomposites of $\mathrm{ZnO}-\mathrm{TiO}_{2}-\mathrm{SiO}_{2}$. Optical Materials, Vol.31, No2, pp.361-365, ISSN: 0925-3467

[6] Bindu Krishnana, Litty Irimpana, V.P.N. Nampoora, V. Kumarc. (2008). Synthesis and nonlinear optical studies of nano ZnO colloids. Physica E, Vol.40, No8, pp. 2787-2790, ISSN: 1386-9477

[7] Yu.N. Kulchin, A.V. Scherbakov, V.P. Dzyuba, S.S. Voznesenskiy, G.T. Mikaelyan. (2008). Nonlinear-optical properties of heterogeneous liquid nanophase composites based on high-energy-gap $\mathrm{Al}_{2} \mathrm{O}_{3}$ nanoparticles. Quantum Electronics, Vol. 38 , No2, pp. 154 - 158, ISSN: 1063-7818 
[8] Yu. N. Kulchin, A. V. Scherbakov, V. P. Dzyuba, S. S. Voznesenskiy. (2009). Interaction of Collinear Light Beams with Different Wavelengths in a heterogeneous liquid-phase nanocomposite. Technical Physics Letters (Rus), Vol.35, No. 7, pp. 640-642, ISSN: 0320-0116

[9] Yu. N. Kulchin, V.P. Dzyuba, A.V. Scherbakov, S.S. Voznesenskiy. (2009). Nonlinear optical interaction of radiation with heterogeneous liquid-phase nanocomposites In: Nonlinear waves (2008), academician Gaponov-Grehov, pp. 361-382, ISBN: 978-5-8048-0069-8, Nizhny Novgorod

[10] Yu.N. Kulchin, V.P. Dzyuba, A.V. Scherbakov. (2009). Optical Transmittance Spectra of Insulator nanoparticles in bulk heterocomposites. Semiconductors (Rus), Vol.43, No. 3, pp. 331-339, ISSN:0015-3222

[11] L.D. Landau, L.D. Lifshitz. (2002). Quantum Mechanics, Fizmatlit, ISBN: 5-9221-0057-2, Moscow

[12] Miheev, A.I. Sidorov. (2004). Optical nonlinearity of nanoparticles of wide-gap semiconductors and insulators in visible and near infrared spectral region. Technical Physics (Rus), Vol.74, No. 6, pp.77-82, ISSN: 0044-4642

[13] Noriyuki Miyata, Masakazu Ichikawa. (2004). Scanningprobe-induced defects in thin $\mathrm{SiO}_{2}$ film on $\mathrm{Si}$ : Comparison with Si clusters. Phys. Rev. B. V.70 No7, pp. 07306-07340

[14] A.I. Ryasnyanskiy, B. Palpant, S. Debrus, U. Pal, A.L. Stepanov. (2009). Nonlinear Optical Properties of Gold Nanoparticles Dispersed in Different Optically Transparent Matrices. Physics of the Solid State, Vol.51, No. 1, pp. 5560, ISSN: 1063-7834

[15] S.I. Pokutnyi. (2006). Absorption and scattering of light by one-particle states of charge carriers in semiconductor quantum dots. Semiconductors (Rus), Vol.40, No. 2, pp. 223-228, ISSN: 0015-3222;

[16] S.I.Pokutnyi. (2013). Absorption of light in positron and electron states in quasi-zero-dimensional nanosystems. Optics, Vol.2, No.4, pp. 47-50.

[17] S.I.Pokutnyi. (2013). Absorption of light at electron states in quasi-zero-dimensional nanosystems. Technical Physics (Rus), Vol.58, No.11, pp. 1661-1664, ISSN: 1063-7842.

[18] S.I.Pokutnyi. (2011). Exciton states in quantum dots. Phys.Express, Vol.1, No.3, pp. 158-168.

[19] S.I.Pokutnyi, (2005). Optical nanolaser heavy hole transitions in quasi - zero - dimensional semiconductors nanosystems. Physics Letters A, V.342, No. 5, pp. 347-352.

[20] S.I.Pokutnyi. (2011). Interband absorption of light in quantum dots. J. Nanoscience Lett., V.1, No. 3, pp.191-198.

[21] S.I.Pokutnyi. (2004). Size quantization Stark effect in semiconductor quantum dots. J. Appl. Phys. V.96, No. 2, pp. $1115-1122$.

[22] S.I.Pokutnyi. (2014). Local electron states in ellipsoidal nanosystems in homogeneous magnetic field. Open Journal of Modern Physics. V. 2, No. 6, pp. 22-29.

[23] Y.R. Shen. (1983). The Principles Of Nonlinear Optics,. John Wiley \& Sons, ISBN: 5-02-014043-0

[24] A.G. Vituhnovsky, A.A. Isaev, V.S. Lebedev. (2008). Light-induced nonlinearity of CdSe/ZNs quantum dots with millisecond relaxation tine. Russian nanotechnologies, Vol. 3, No. 11-12, pp. 110-117, ISSN: 1992-7223 